\title{
Flow-induced instabilities of springs-mounted plates in viscous flows: a global stability approach
}

\author{
Johann Moulin ${ }^{1, \text { a) }}$ and Olivier Marquet ${ }^{1}$ \\ ONERA-DAAA, 8 rue des Vertugadins, 92190 Meudon, France
}

(Dated: 1 March 2021)

The linear stability of a typical aeroelastic section, consisting in a rectangular plate mounted on flexion and torsion springs, is revisited here for low-Reynolds-number incompressible flows. By performing global stability analyses of the coupled fluid-solid equations, we find four types of unstable modes related to different physical instabilities and classically investigated with separate flow models: coupled-mode flutter, single-mode flutter and static divergence at high reduced velocity $U^{*}$ and vortex-induced vibrations at low $U^{*}$. Neutral curves for these modes are presented in the parameter space composed of the solid-to-fluid mass ratio and the reduced velocity. Interestingly, the flutter mode is seen to restabilize for high reduced velocities thus leading to a finite extent flutter region, delimited by low $U^{*}$ and high- $U^{*}$ boundaries. At a particular low mass ratio, both boundaries merge such that no flutter instability is observed for lower mass ratios. The effect of the Reynolds number is then investigated, indicating that the high- $U^{*}$ restabilization strongly depends on viscosity. The global stability results are compared to a statically calibrated Theodorsen model: if both approaches converge in the high mass ratio limit, they significantly differ at lower mass ratios. In addition, the Theodorsen model fails to predict the high- $U^{*}$ restabilization of the flutter mode.

\section{INTRODUCTION}

Flow-induced vibrations of coupled fluid-solid systems are ubiquitous in engineering. One of their most classical illustrations is probably the coupled-mode flutter of aircraft wings at high speed ${ }^{1,2}$. This phenomenon designates vibrations that develop around a steady angle of attack close to zero, through the flow-induced coupling of at least two natural modes of the wing (e.g. flexion and torsion). When the steady angle of attack is increased, the flow tends to stall ${ }^{3,4}$, leading to a different type of flutter, usually involving one natural solid mode only and referred to as stall flutter ${ }^{5-7}$. When the flow is fully separated at post-stall angles of attack, the airfoil can be viewed as a bluff body. The well-known vortexshedding flow-instability may then interact with a natural mode of the solid with similar frequency, leading to the so-called vortex-induced vibrations ${ }^{8}$ (VIV). Historically, these different types of vibrations were mostly observed on large-scale objects surrounded by high-speed flows - plane wings, helicopter blades, tall buildings, bridges, etc — where they put at risk the integrity of the structure. In the recent years however, several new and smaller-scale applications have been proposed that take advantage of flow-induced vibrations for energy harvesting purposes ${ }^{9}$ or for improving the performance of small-scale mixers ${ }^{10,11}$ and heat exchangers ${ }^{12,13}$. For these applications, the objective is generally to design the system so as to maximize the vibrations. This can be done either experimentally ${ }^{14-20}$ or numerically by running multiple simulations of the coupled fluid-solid nonlinear equations ${ }^{18,21-26}$. In order to minimize the experimental or computational effort, it is however interesting

\footnotetext{
a) johann.moulin@onera.fr
}

to first delimit the regions of the parameter space where flow-induced vibrations will occur, using linear stability analysis $^{21,22}$.

As coupled-mode flutter, stall flutter or vortex-induced vibrations originate from different physical mechanisms, their respective description usually involves separate flow models.

For coupled-mode flutter, a classical choice is to resort to potential flow theories ${ }^{15,21,22}$, such as the Theodorsen model $^{27}$, that have encountered a great success for the computation of aeroelastic stability of aircraft ${ }^{28}$. However, in contrast to aircraft applications that naturally yield high-Reynolds-number flows, energy harvesting or micro-scale mixing typically involve low-to-moderate Reynolds numbers ${ }^{10,11,18,21-26}$, ranging from $\mathcal{R} e=10^{1}$ to $\mathcal{R} e=10^{5}$. For these Reynolds numbers, the validity of the potential flow assumption must be questioned, as shown by Bruno and Fransos ${ }^{29}$ or Brunton and Rowley 30 for the case of thin plates forced in heaving or pitching motions. As these studies were primarily interested in accurately predicting the aerodynamic forces consecutive to forced motions, the question of the impact of low-tomoderate Reynolds numbers on the self-sustained flutter instability was not investigated. This naturally motivates a study discussing coupled-mode flutter in viscous flows. Such an interest is further justified from the perspective of modern flying devices, like nano- and micro- aerial vehicles that also operate at low-to-moderate Reynolds numbers ${ }^{31}$.

In the case of stall flutter and vortex-induced vibrations, large regions of flow separation are involved, thus implying that flow models based on potential theories are unsuitable. For the particular case of a stall flutter involving only a transverse motion of the structure, and where vibrations are slow enough, so-called quasi-steady flow models may be used ${ }^{32}$. The advantage of the latter is that they only require knowing the steady aerody- 
namic loads at various angles of attack. For all situations where the quasi-steady approaches cannot be used - e.g. vortex-induced vibrations — one may either develop tailored semi-empirical reduced-order models ${ }^{33,34}$ or rather retain the full complexity of the time-dependent nonlinear Navier-Stokes equations ${ }^{23-25,35}$, but at the cost of an intensive computational effort.

When linear stability is the primary interest, a path of intermediate cost may be taken where a full NavierStokes flow model is retained, but in a linearized form. This approach, often referred to as global stability analysis, was originally used to investigate the linear stability of flows around fixed objects by computing the leading eigenvalues of the Navier-Stokes Jacobian matrix ${ }^{36,37}$. This framework was first extended to fluid-solid interaction problems to investigate the vortex-induced vibrations of spring-mounted rigid cylinders in low-Reynoldsnumber flows ${ }^{38-41}$. More recently, it was used to predict the transonic shock buffeting instability of springmounted airfoils ${ }^{42,43}$. Other possible applications are the study of path instabilities of freely falling or rising rigid objects $^{44-46}$ or of the onset of pitch-oscillations on airfoils at transitional Reynolds numbers ${ }^{47}$. These analyses were recently extended to account for deformable structures governed by elastic laws ${ }^{48-51}$ or to address the fluid-solid stability analysis of self-propelled flapping foils ${ }^{52}$. To our knowledge however, they have not been used to investigate the instabilities occurring on spring-mounted slender bodies that are typical of coupled-mode or stall flutter.

In the present study, we thus aim at investigating, in low-Reynolds number flows, the flow-induced instabilities that occur on a typical aeroelastic section ${ }^{2}$ consisting in a heaving and pitching spring-mounted rigid plate. Our goal is to demonstrate the use of global fluid-solid linear stability analysis for providing a parametric exploration of this configuration by varying the reduced velocity, the solid-to-fluid mass ratio, the Reynolds number and the steady angle of attack. The paper is organized as follows. In section II, we briefly introduce the springmounted plate model and the global fluid-solid stability approach. In section III, we exhibit the different types of instabilities that appear at zero steady angle of attack, depending on the reduced velocity and solid-to-fluid mass ratio. We then explore the effect of the Reynolds number and present a comparison of our results with a calibrated version of the Theodorsen model. In section IV, we finally explore the effect of increasing the steady angle of attack on the flutter instability.

\section{PROBLEM SETTINGS AND METHODS}

We investigate the dynamics of a rigid plate of chord $\tilde{c}$, thickness $\tilde{e}$ and $\operatorname{span} \tilde{s}$. The two-dimensional section of the plate is almost rectangular with small rounded corners of radius $\tilde{e} / 10$. Its chord-to thickness ratio is $\tilde{c} / \tilde{e}=20$ similarly to previous investigations ${ }^{14,19}$. The mass of the plate and its moment of inertia at the center

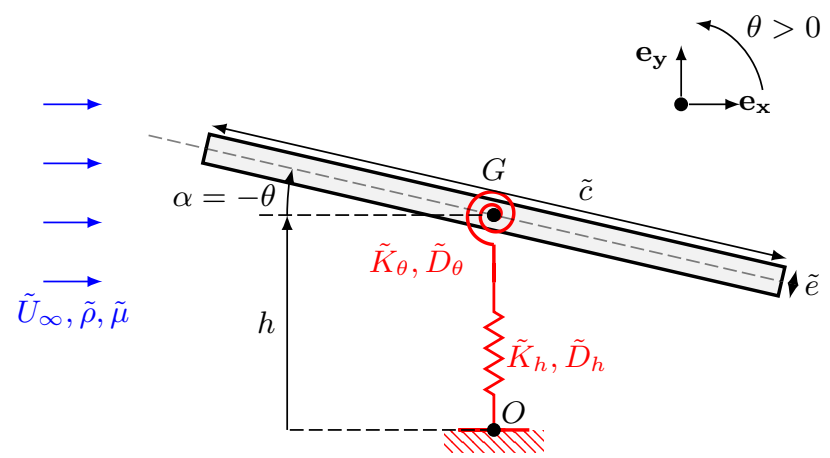

FIG. 1. "Typical section" model.

of mass $G$ are denoted $\tilde{m}$ and $\tilde{I}_{G}$, respectively. This plate is immersed in a fluid of density $\tilde{\rho}$ and dynamic viscosity $\tilde{\mu}$, with a uniform upstream velocity $\tilde{U}_{\infty}$. Assuming the span is much larger than the chord, i.e. $\tilde{s}>>\tilde{c}$, the dynamics of the plate may be modelled by the typical aeroelastic section ${ }^{2}$ sketched in fig. 1. Heaving and pitching springs of stiffness (resp. damping) $\tilde{K}_{h}$ (resp. $\tilde{D}_{h}$ ) and $\tilde{K}_{\theta}$ (resp. $\tilde{D}_{\theta}$ ) are attached to the twodimensional section at the so-called elastic center. In the present study, the latter is identical to the center of mass $G$ and we refer, for example, to Peng and Zhu ${ }^{21}$ for an investigation of the effect of its position on the dynamics.

All quantities presented above and noted with the symbol $\sim$ are dimensional variables. In this work however, we will rather use non-dimensional variables, that we note without this symbol. The non-dimensionalization is performed using $\tilde{c}$ as a characteristic length, $\tilde{c} / \tilde{U}_{\infty}$ as a characteristic time and $\tilde{\rho} \tilde{c}^{2}$ as a characteristic mass (per unit span). The seven non-dimensional parameters listed in table I then govern the dynamics of the typical section interacting with the surrounding incompressible flow. Some of them involve only fluid (resp. solid) dimensional quantities and are thus labelled as fluid (resp. solid) non-dimensional parameters. Others involve both fluid and solid quantities and are thus referred to as coupling parameters. The only fluid parameter is the Reynolds number defined as $\mathcal{R} e=\tilde{\rho} \tilde{U}_{\infty} \tilde{c} / \tilde{\mu}$. Four solid parameters describe the spring-mounted plate: $\Omega=\tilde{\omega}_{0, h} / \tilde{\omega}_{0, \theta}$ is the ratio between the natural heaving and pitching frequencies of the plate, respectively defined as $\tilde{\omega}_{0, h}=\sqrt{\tilde{K}_{h} / \tilde{m}}$ and $\tilde{\omega}_{0, \theta}=\sqrt{\tilde{K}_{h} / \tilde{I}_{G}} ; \zeta_{h}$ and $\zeta_{\theta}$ are the non-dimensional heaving and pitching damping ratios; $r_{\theta}=\sqrt{\tilde{I}_{G} /\left(\tilde{m} \tilde{c}^{2}\right)}$ is the non-dimensional radius of gyration. Finally, two parameters control the fluid-solid coupling: the solid-to-fluid mass ratio $m=\tilde{m} /\left(0.5 \tilde{\rho} \tilde{c}^{2} \tilde{s}\right)$ and the reduced velocity $U^{*}=\tilde{U}_{\infty} /\left(\tilde{c} \tilde{\omega}_{\theta}\right)$ that is also equal to the inverse the non-dimensional pitching frequency $U^{*}=1 / \omega_{\theta}$.

In the present study, the four solid parameters are kept fixed to the values reported in table I. Though not strictly equal, the values of $\Omega, \zeta_{h}$ and $\zeta_{\theta}$ are inspired from the 
set-up of Amandolese, Michelin, and Choquel ${ }^{14}$. The radius of gyration is evaluated under the hypothesis of a rectangular cross-section with homogeneous solid density, yielding $r_{\theta}{ }^{2}=(1 / 12)\left(1+(\tilde{e} / \tilde{c})^{2}\right)$. The fluid and coupling parameters are varied in the ranges reported in table I. Low Reynolds numbers are explored in the range $10^{1} \leq \mathcal{R} e \leq 10^{4}$, which contains the values used in several previous works on energy harvesting devices ${ }^{18,21-25}$. Our study however excludes the moderate Reynolds number range $10^{4} \leq \mathcal{R} e \leq 10^{5}$ where transitional effects come into play and trigger specific fluid-solid instabilities ${ }^{53-55}$. This regime indeed requires the use of turbulence models with adequate transition prediction that are out of the scope of the present work. For a plate made of a given material, the mass ratio $m$ may significantly vary due to variation of the fluid density. For example, in the experimental configuration by Amandolese, Michelin, and Choquel ${ }^{14}$, a steel plate immersed in air flow corresponds to $m \sim 1800$, while a steel airfoil immersed in water flow in the experimental set-up by Boudreau et al. ${ }^{20}$ now corresponds to $m \sim 1.7$. Low values of the mass ratio, typically of the order $m \sim 10$, can be obtained in air flows with very light materials, such as balsa wood $^{56}$. In order to explore these limit scenarios and the transition between them, we consider a large range of mass ratios, $10^{0} \leq m \leq 10^{4}$. Finally, the reduced velocity varies roughly between $10^{-2}$ and $10^{1}$ which allows visiting both low- $U^{*}$ instabilities like vortex-induced vibrations and higher- $U^{*}$ instabilities like flutter and divergence.

\section{A. Mathematical models}

The motion of the plate is described in the laboratory frame of reference $\left(O, \mathbf{e}_{\mathbf{x}}, \mathbf{e}_{\mathbf{y}}\right)$ with two degrees of freedom: the non-dimensional displacement $h(t)$ (in the direction $\mathbf{e}_{\mathbf{y}}$ ) and the pitching angle $\theta(t)$ (oriented counterclockwise, i.e. opposite to the angle of attack $\alpha$, see fig. 1). They are governed by the following coupled system of linear equations:

$$
\begin{array}{r}
\frac{\mathrm{d}^{2} h}{\mathrm{~d} t^{2}}+2 \zeta_{h}\left(\frac{\Omega}{U^{*}}\right) \frac{\mathrm{d} h}{\mathrm{~d} t}+\left(\frac{\Omega}{U^{*}}\right)^{2} h=\frac{1}{m}\left(\mathcal{C}_{L}(\mathbf{U}, P, \theta)\right. \\
\left.+\mathcal{C}_{F_{\text {ext }}}\right) \\
\frac{\mathrm{d}^{2} \theta}{\mathrm{d} t^{2}}+2 \zeta_{\theta}\left(\frac{1}{U^{*}}\right) \frac{\mathrm{d} \theta}{\mathrm{d} t}+\left(\frac{1}{U^{*}}\right)^{2} \theta=\frac{1}{m r_{\theta}{ }^{2}}\left(\mathcal{C}_{M}(\mathbf{U}, P)\right. \\
\left.+\mathcal{C}_{M_{\text {ext }}}\right)
\end{array}
$$

where the ratio $\Omega / U^{*}$ is the (non-dimensional) natural heaving frequency $\tilde{\omega}_{0, h}$ while $1 / U^{*}$ is the (nondimensional) natural pitching frequency $\tilde{\omega}_{0, \theta}$. The above equations are two damped harmonic oscillators forced by the aerodynamic lift $\mathcal{C}_{L}=\tilde{L} /\left(0.5 \tilde{\rho} \tilde{U}_{\infty}^{2} \tilde{c} \tilde{s}\right)$ (with $\tilde{L}$ the lift force) and moment $\mathcal{C}_{M}=\tilde{M} /\left(0.5 \tilde{\rho} \tilde{U}_{\infty}^{2} \tilde{c}^{2} \tilde{s}\right)$ (with $\tilde{M}$ the aerodynamic moment) coefficients that depend on

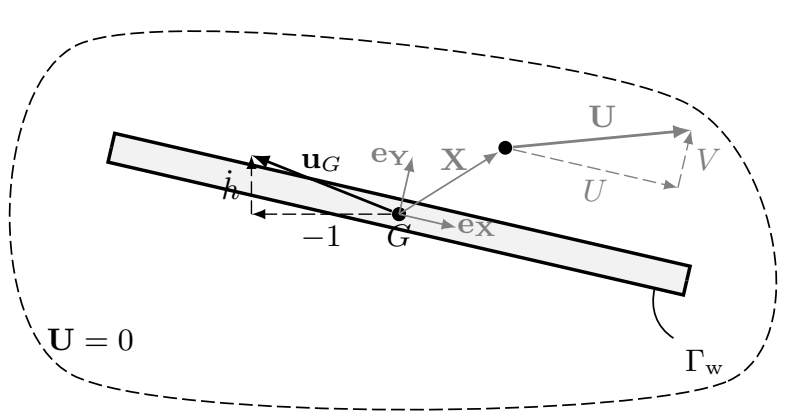

FIG. 2. Illustration of the quantities describing the (nondimensional) Navier-Stokes formalism by Mougin and Magnaudet $^{57}$.

the flow velocity $\mathbf{U}$ and pressure $P$, as further detailed in eq. (5). These aerodynamics coefficients are responsible for the coupling between the heaving and pitching components of the solid motion. In addition, a constant external force $\mathcal{C}_{F_{\text {ext }}}$ and moment $\mathcal{C}_{M_{\text {ext }}}$ are taken into account, their use being specified in section II B.

The two-dimensional incompressible flow may be described by the pressure field $p(\mathbf{x}(t), t)$ and the velocity field vector $\mathbf{u}(\mathbf{x}(t), t)=(u, v)^{\mathrm{T}}$ expressed in fixed laboratory frame $\left(O, \mathbf{e}_{\mathbf{x}}, \mathbf{e}_{\mathbf{y}}\right)$. These fields then satisfy the incompressible Navier-Stokes equations in a spatial domain that depends on time due to the plate's motion, as emphasized by the time dependency of the spatial coordinate $\mathbf{x}(t)=(x(t), y(t))^{\mathrm{T}}$. In order to avoid the deformation of the fluid domain, we adopt the formalism proposed by Mougin and Magnaudet ${ }^{57}$ where the spatial positions, denoted $\mathbf{X}=(X, Y)$, are expressed in the frame $\left(G(t), \mathbf{e}_{\mathbf{X}}(t), \mathbf{e}_{\mathbf{Y}}(t)\right)$ that is attached to the center of mass $G(t)$ and that rotates with the principal axes of the plate (see fig. 2). Using these spatial coordinates, the fluid domain becomes time-independent. The absolute velocity vector written in the rotating axes $\left(\mathbf{e}_{\mathbf{X}}(t), \mathbf{e}_{\mathbf{Y}}(t)\right)$ is then denoted $\boldsymbol{U}(\mathbf{X}, t)=(U, V)^{T}$ and may be linked to u by:

$$
\begin{gathered}
\mathbf{U}(\mathbf{X}, t)=\mathbf{R}(\theta(t))^{\mathrm{T}} \mathbf{u}(\mathbf{x}(t), t) \\
\text { where } \quad \mathbf{R}(\theta)=\left(\begin{array}{cc}
\cos (\theta) & -\sin (\theta) \\
\sin (\theta) & \cos (\theta)
\end{array}\right)
\end{gathered}
$$

is the rotation matrix of angle $\theta$. We also denote the pressure field depending on $\mathbf{X}$ as $P(\mathbf{X}, t)=p(\mathbf{x}(t), t)$. With these specific choices, the incompressible NavierStokes equations read $^{57}$ :

$$
\frac{\partial \mathbf{U}}{\partial t}+\dot{\theta} \mathbf{e}_{\mathbf{z}} \wedge \mathbf{U}+\nabla \mathbf{U}(\mathbf{U}-\mathbf{W})-\nabla \cdot \boldsymbol{\sigma}(\mathbf{U}, P)=0
$$

$$
\nabla \cdot \mathbf{U}=0
$$

where $\boldsymbol{\sigma}(\mathbf{U}, P)=-P \mathbf{I}+\mathcal{R} e^{-1}\left(\nabla \mathbf{U}+\nabla \mathbf{U}^{\mathrm{T}}\right)$ is the Cauchy stress tensor, and, with a slight abuse of notation, $\mathbf{e}_{\mathbf{z}} \wedge \mathbf{U}=(-V, U)^{\mathrm{T}}$. Compared to the classical 
TABLE I. Non-dimensional parameters governing the typical aeroelastic section ordered as the fluid parameter $(\mathcal{R} e)$, solid parameters $\left(\Omega, \zeta_{h}, \zeta_{\theta}\right.$ and $\left.r_{\theta}\right)$ and coupling parameters $\left(m\right.$ and $\left.U^{*}\right)$.

\begin{tabular}{lccccccc}
\hline \hline Parameter & $\mathcal{R} e$ & $\Omega$ & $\zeta_{h}$ & $\zeta_{\theta}$ & $r_{\theta}$ & $m$ & $U^{*}$ \\
\hline \multirow{2}{*}{ Definition } & $\frac{\tilde{\rho} \tilde{U}_{\infty} \tilde{c}}{\tilde{\mu}}$ & $\sqrt{\frac{\tilde{K}_{h} / \tilde{m}}{\tilde{K}_{\theta} / \tilde{I}_{G}}}$ & $\frac{\tilde{D}_{h}}{2 \sqrt{\tilde{m} \tilde{K}_{h}}}$ & $\frac{\tilde{D}_{\theta}}{2 \sqrt{\tilde{I}_{G} \tilde{K}_{\theta}}}$ & $\sqrt{\frac{\tilde{I}_{G}}{\tilde{m} \tilde{c}^{2}}}$ & $\frac{\tilde{m}}{0.5 \tilde{\rho} \tilde{c}^{2} \tilde{s}}$ & $\frac{\tilde{U}_{\infty}}{\tilde{c} \sqrt{\tilde{K}_{\theta} / \tilde{I}_{G}}}$ \\
Values & $10^{1}-10^{4}$ & 0.8 & 0 & 0.05 & 0.29 & $10^{0}-10^{4}$ & $10^{-2}-10^{1}$ \\
\hline \hline
\end{tabular}

formulation of the Navier-Stokes equations, we note the Coriolis-like acceleration term that depends on the angular velocity $\dot{\theta}=\mathrm{d} \theta / \mathrm{d} t$ of the plate, as well as the modification of the convective velocity by the so-called grid velocity $\mathbf{W}$, often introduced in the Arbitrary Lagrangian Eulerian framework ${ }^{58}$. In the rotating axes, this grid velocity reads

$$
\mathbf{W}(\theta, \dot{h}, \dot{\theta} ; \mathbf{X})=\mathbf{R}(\theta)^{\mathrm{T}} \mathbf{u}_{\mathrm{G}}+\dot{\theta}\left(\mathbf{e}_{\mathbf{z}} \wedge \mathbf{X}\right)
$$

with $\dot{h}=\mathrm{d} h / \mathrm{d} t$ and where $\mathbf{u}_{\mathrm{G}}=(-1, \dot{h})^{\mathrm{T}}$ is the (nondimensional) velocity of the center of mass $G$, written in the fixed laboratory axes $\left(\mathbf{e}_{\mathbf{x}}, \mathbf{e}_{\mathbf{y}}\right)$. In particular, the plates moves at the velocity -1 along the direction $\mathbf{e}_{\mathbf{x}}$ (see fig. 2). The second term in the above expression is the angular velocity at point $\mathbf{X}$. When evaluating this grid velocity at points $\mathbf{X}_{w}$ on the plate $\Gamma_{w}$, we recover the solid velocity expressed in the rotating axes. The equality of the fluid and solid velocities at this interface thus reads:

$$
\mathbf{U}\left(\mathbf{X}_{\mathrm{w}}\right)=\mathbf{W}\left(\theta, \dot{h}, \dot{\theta} ; \mathbf{X}_{\mathrm{w}}\right) \quad \mathbf{X}_{\mathrm{w}} \in \Gamma_{\mathrm{w}}
$$

The far-field flow velocity is $\mathbf{U}(\mathbf{X}, t)=0$. Finally, the lift coefficient $\mathcal{C}_{L}$ and moment coefficient $\mathcal{C}_{M}$ evaluated at point $G$, that appear in the right-hand side of the solid equations (1) can now be explicitly written as:

$$
\begin{aligned}
& \mathcal{C}_{L}(\mathbf{U}, P, \theta)=2\{\left.\mathbf{R}(\theta) \int_{\Gamma_{\mathrm{w}}} \boldsymbol{\sigma}(\mathbf{U}, P) \mathbf{N} d \mathbf{X}_{\mathrm{w}}\right\} \cdot \mathbf{e}_{\mathbf{y}} \\
& \mathcal{C}_{M}(\mathbf{U}, P)=2 \int_{\Gamma_{\mathrm{w}}}\left\{X_{\mathrm{w}}(\boldsymbol{\sigma}(\mathbf{U}, P) \mathbf{N})_{Y}\right. \\
&\left.-Y_{\mathrm{w}}(\boldsymbol{\sigma}(\mathbf{U}, P) \mathbf{N})_{X}\right\} d \mathbf{X}_{\mathrm{w}}
\end{aligned}
$$

where $\mathbf{N}$ is the (outward) unit vector normal to the solid (written in the rotating axes). Note in particular the term between curly brackets in eq. (5a) where the fluid force evaluated in the rotating axes is projected back onto the laboratory axes using the rotation matrix, before extracting the $\mathbf{e}_{\mathbf{y}}$ component via the scalar product.

In the following, the equations (1) to (5) governing the fluid-solid interaction are formally written as the firstorder-in-time evolution equation:

$$
\left(\begin{array}{cc}
\mathscr{M}_{\mathrm{s}} & 0 \\
0 & \mathscr{M}_{\mathrm{f}}
\end{array}\right) \frac{\partial}{\partial t}\left(\begin{array}{c}
\mathbf{q}_{\mathrm{s}} \\
\mathbf{q}_{\mathrm{f}}
\end{array}\right)+\left(\begin{array}{c}
\mathscr{R}_{\mathrm{s}}\left(\mathbf{q}_{\mathrm{s}}, \mathbf{q}_{\mathrm{f}}\right) \\
\mathscr{R}_{\mathrm{f}}\left(\mathbf{q}_{\mathrm{s}}, \mathbf{q}_{\mathrm{f}}\right)
\end{array}\right)=0
$$

where $\mathbf{q}_{\mathrm{s}}=(h, \theta, \dot{h}, \dot{\theta})^{\mathrm{T}}$ and $\mathbf{q}_{\mathrm{f}}=(\mathbf{U}, P)^{\mathrm{T}}$ gather the solid and flow variables, respectively. The detailed expressions of the solid $\mathscr{M}_{\mathrm{s}}$ and fluid $\mathscr{M}_{\mathrm{f}}$ mass matrices, as well as the solid $\mathscr{R}_{\mathrm{s}}$ and fluid $\mathscr{R}_{\mathrm{f}}$ residual vectors are given in appendix A.

\section{B. Global fluid-solid linear stability analysis}

We investigate the linear stability of steady solutions, denoted $\left(\mathbf{q}_{\mathrm{s}}^{0}, \mathbf{q}_{\mathrm{f}}^{0}\right)^{\mathrm{T}}$, that are time-independent solutions of eq. (6), by superimposing unsteady perturbations as:

$$
\left(\begin{array}{c}
\mathbf{q}_{\mathrm{s}}(t) \\
\mathbf{q}_{\mathrm{f}}(\mathbf{X}, t)
\end{array}\right)=\left(\begin{array}{c}
\mathbf{q}_{\mathrm{s}}^{0} \\
\mathbf{q}_{\mathrm{f}}^{0}(\mathbf{X})
\end{array}\right)+\epsilon\left(\begin{array}{c}
\mathbf{q}_{\mathrm{s}}^{1}(t) \\
\mathbf{q}_{\mathrm{f}}^{1}(\mathbf{X}, t)
\end{array}\right) \quad \epsilon \ll 1 .
$$

The infinitesimal fluid-solid perturbation $\left(\mathbf{q}_{\mathrm{s}}^{1}, \mathbf{q}_{\mathrm{f}}^{1}\right)^{\mathrm{T}}$ is further decomposed in the form of global modes ${ }^{36}$ :

$$
\left(\begin{array}{c}
\mathbf{q}_{\mathbf{s}}^{1}(t) \\
\mathbf{q}_{\mathbf{f}}^{1}(\mathbf{X}, t)
\end{array}\right)=\frac{1}{2}\left(\begin{array}{c}
\widehat{\mathbf{q}_{\mathrm{s}}} \\
\widehat{\mathbf{q}_{\mathrm{f}}}(\mathbf{X})
\end{array}\right) e^{\sigma t}+\text { c.c. }
$$

where c.c. is the abbreviation for complex conjugate and $\sigma=\lambda+\mathbf{i} \omega$ is a complex number whose real (resp. imaginary) part is the growth rate (resp. frequency) of the complex-valued fluid-solid mode $\left(\widehat{\mathbf{q}_{s}}, \widehat{\mathbf{q}}_{\mathrm{f}}\right)^{\mathrm{T}}$. Inserting the above decomposition in (6) yields that $\left(\mathbf{q}_{\mathrm{s}}^{0}, \mathbf{q}_{\mathrm{f}}^{0}\right)^{\mathrm{T}}$ is solution of the steady nonlinear problem:

$$
\left(\begin{array}{c}
\mathscr{R}_{\mathrm{s}}\left(\mathbf{q}_{\mathrm{s}}^{0}, \mathbf{q}_{\mathrm{f}}^{0}\right) \\
\mathscr{R}_{\mathrm{f}}\left(\mathbf{q}_{\mathrm{s}}^{0}, \mathbf{q}_{\mathrm{f}}^{0}\right)
\end{array}\right)=0
$$

and that $\left(\sigma,\left(\widehat{\mathbf{q}_{\mathrm{s}}}, \widehat{\mathbf{q}_{\mathrm{f}}}\right)^{\mathrm{T}}\right)$ are eigenpairs of the following eigenproblem:

$$
\sigma\left(\begin{array}{cc}
\mathscr{M}_{\mathrm{s}} & 0 \\
0 & \mathscr{M}_{\mathrm{f}}
\end{array}\right)\left(\begin{array}{c}
\widehat{\mathbf{q}}_{\mathrm{s}} \\
\widehat{\mathbf{q}}_{\mathrm{f}}
\end{array}\right)+\left(\begin{array}{cc}
\mathscr{J}_{\mathrm{ss}} & \mathscr{J}_{\mathrm{sf}} \\
\mathscr{J}_{\mathrm{fs}} & \mathscr{J}_{\mathrm{ff}}
\end{array}\right)\left(\begin{array}{l}
\widehat{\mathbf{q}}_{\mathrm{s}} \\
\widehat{\mathbf{q}}_{\mathrm{f}}
\end{array}\right)=0
$$

The operators $\mathscr{J}_{\alpha \beta}=\partial \mathscr{R}_{\alpha} /\left.\partial \mathbf{q}_{\beta}\right|_{\left(\mathbf{q}_{\mathrm{s}}^{0}, \mathbf{q}_{\mathrm{f}}^{0}\right)}$, with $\alpha, \beta=$ $\{\mathrm{s}, \mathrm{f}\}$, define by blocks the fluid-solid Jacobian operator. They implicitly depend on the steady solution as specified in the detailed expressions provided in appendix A. The long term stability of steady solutions is assessed by scrutinizing the eigenvalue of largest real part. If all 
eigenvalues have negative real parts $(\lambda<0)$ the system is stable in the sense that any infinitesimal perturbation will eventually decay to zero. If at least one eigenvalue has positive real part $(\lambda>0)$ then the system is unstable. The case $\lambda=0$ describes a situation where a perturbation will neither be amplified nor damped, and is said neutrally stable.

a. Method for computing steady fluid-solid solutions In the steady solution $\left(\mathbf{q}_{\mathrm{s}}^{0}, \mathbf{q}_{\mathrm{f}}^{0}\right)^{\mathrm{T}}$ of $(7)$, the heaving $h^{0}$ and pitching $\theta^{0}$ components are unknowns of the fluidsolid problem. In this work however, we choose to impose the value of $h^{0}$ and $\theta^{0}$ as parameters in order to investigate directly the effect of the steady angle of attack on the fluid-solid instabilities. More precisely, we use $h^{0}=0$ and $\theta^{0}=-\alpha^{0}$ with $\alpha^{0}$ the steady angle of attack (oriented positive clockwise). The solid part $\mathbf{q}_{\mathrm{s}}^{0}$ of the steady solution is thus known in advance as $\mathbf{q}_{\mathrm{s}}^{0}=\left(0,-\alpha^{0}, 0,0\right)^{\mathrm{T}}$. The fluid part $\mathbf{q}_{\mathrm{f}}^{0}$ of the steady solution is then obtained by solving the purely fluid steady problem:

$$
\mathscr{R}_{\mathrm{f}}\left(\mathbf{q}_{\mathrm{s}}^{0}, \mathbf{q}_{\mathrm{f}}^{0}\right)=0
$$

Note that the resulting fluid-solid solution $\left(\mathbf{q}_{\mathrm{s}}^{0}, \mathbf{q}_{\mathrm{f}}^{0}\right)^{\mathrm{T}}$ is a solution of the original steady problem (7) by opportunely choosing the exterior force and moment coefficients, $\mathcal{C}_{F_{\text {ext }}}$ and $\mathcal{C}_{M_{\text {ext }}}$, so as to enforce the steady solid momentum balance (1):

$$
\begin{aligned}
\mathcal{C}_{F_{\text {ext }}} & =-\mathcal{C}_{L}\left(\mathbf{U}^{0}, P^{0},-\alpha^{0}\right) \\
\mathcal{C}_{M_{\text {ext }}} & =m r_{\theta}{ }^{2}\left(\frac{1}{U^{*}}\right)^{2}\left(-\alpha^{0}\right)-\mathcal{C}_{M}\left(\mathbf{U}^{0}, P^{0}\right)
\end{aligned}
$$

It is important to notice here that $\mathcal{C}_{F_{\text {ext }}}$ and $\mathcal{C}_{M_{\text {ext }}}$ are constant exterior forces applied to eq. (1) as they depend only on the steady solution and not on the unknown timedependent solution $\left(\mathbf{q}_{\mathrm{s}}(t), \mathbf{q}_{\mathrm{f}}(\mathbf{X}, t)\right)^{\mathrm{T}}$. They thus play no role in the linear stability analysis as can be seen in the fluid-solid Jacobian expressions of appendix A where they do not appear. Overall, by using such a procedure, the steady solution is made independent on the solid $\left(\Omega, \zeta_{h}, \zeta_{\theta}, r_{\theta}\right)$ and coupling $\left(U^{*}, m\right)$ non-dimensional parameters and only depends on the fluid non-dimensional parameter - here the Reynolds number $\mathcal{R} e$ - and the chosen steady angle of attack $\alpha^{0}$.

$b$. Method for determining neutral curves To delimit stable $(\lambda<0)$ from unstable $(\lambda>0)$ regions in a parameter space, one could discretize the latter and solve the eigenproblem (8) for each discrete point. A more efficient method consists in considering the critical values of these parameters as unknowns of a problem that incorporates the criticality condition $\lambda=0$. For a two-dimensional parameter space, for instance composed of the mass ratio $m$ and reduced velocity $U^{*}$ (see section IIIC), the so-called neutral curve is defined as the critical velocity $U_{c}^{*}$ as a function of the mass ratio $m$ and is obtained as follows. The growth rate being, by definition, equal to zero on the neutral curve, the critical eigenmode $\left(\widehat{\mathbf{q}}_{\mathrm{s}}{ }^{c}, \widehat{\mathbf{q}}_{\mathrm{f}}^{c}\right)^{\mathrm{T}}$ and frequency $\omega_{\mathrm{c}}$ satisfy:

$$
\mathrm{i} \omega_{\mathrm{c}}\left(\begin{array}{cc}
\mathscr{M}_{\mathrm{s}} & 0 \\
0 & \mathscr{M}_{\mathrm{f}}
\end{array}\right)\left(\begin{array}{c}
\widehat{\mathbf{q}}_{\mathrm{s}}{ }^{c} \\
\widehat{\mathbf{q}}_{\mathrm{f}}
\end{array}\right)+\left(\begin{array}{cc}
\mathscr{J}_{\mathrm{ss}}\left(U_{c}^{*}\right) & \mathscr{J}_{\mathrm{sf}} \\
\mathscr{J}_{\mathrm{fs}} & \mathscr{J}_{\mathrm{ff}}
\end{array}\right)\left(\begin{array}{c}
\widehat{\mathbf{q}}_{\mathrm{s}} \\
\widehat{\mathbf{q}}_{\mathrm{f}}
\end{array}\right)=0
$$

where we recall the dependency of the solid Jacobian matrix on the critical velocity $U_{c}^{*}$. The latter being here an additional (real-valued) unknown of the problem instead of a parameter, an additional (real-valued) scalar condition is imposed, formally written as:

$$
\mathscr{C}\left(\widehat{\mathbf{q}}_{\mathrm{s}}^{c}, \widehat{\mathbf{q}}^{c}, \omega_{\mathrm{c}}\right)=0
$$

In practice, we only introduce this additional condition for dynamic $\left(\omega_{c} \neq 0\right)$ instabilities. The phase of the critical eigenmode is fixed by imposing that the real part of the heaving degree of freedom is zero, i.e. $\mathscr{C}\left(\widehat{\mathbf{q}}_{\mathrm{s}}{ }^{c}, \widehat{\mathbf{q}}_{\mathrm{f}}^{c}, \omega_{\mathrm{c}}\right)=\mathfrak{R e}\left(\widehat{h}^{c}\right)=0$. The arbitrary amplitude of the critical eigenmode is finally imposed using the following normalization condition:

$$
\left(\left(\widehat{\mathbf{q}}_{\mathrm{s}}^{c}\right)^{\mathrm{H}},\left(\widehat{\mathbf{q}}_{\mathrm{f}}^{c}\right)^{\mathrm{H}}\right)\left(\begin{array}{cc}
\mathscr{M}_{\mathrm{s}} & 0 \\
0 & \mathscr{M}_{\mathrm{f}}
\end{array}\right)\left(\begin{array}{c}
{\widehat{\mathbf{q}_{\mathrm{s}}}}^{c} \\
{\widehat{\mathbf{q}_{\mathrm{f}}}}^{c}
\end{array}\right)-1=0
$$

The coupled system of equations (10), (11) and (12) is nonlinear due to the dependence of the Jacobian operator in $U_{c}^{*}$ and to the presence of $\omega_{c}$ in front of the mass matrix term in eq. (10). A Newton method is used to solve iteratively this system of equations. Thus we obtain the critical velocity $U_{c}^{*}$ (as well as the critical frequency $\omega_{\mathrm{c}}$ and eigenmode $\left.\widehat{\mathbf{q}}_{\mathrm{s}}^{c}, \widehat{\mathbf{q}}_{\mathrm{f}}^{c}\right)$ for a fixed mass ratio $m$ (the remaining parameters being also imposed). From this point of the neutral curve, we can efficiently determine the entire curve by implementing a continuation algorithm, such as the pseudo-arclength method (see the book by Govaerts ${ }^{59}$ ). This method allows, in particular, to follow the neutral curve around turning points (see for instance fig. 8).

c. Numerical method The Navier-Stokes equations are discretized in space with a SUPG-stabilized finite element method ${ }^{60}$, implemented using the FreeFEM language $^{61}$. A Newton method is then used to solve the steady equation (9) and the shift-and-invert method is used to compute the largest eigenvalues of the eigenproblem (8). We refer to Moulin, Jolivet, and Marquet $^{62}$ for a detailed description of these numerical methods in a purely aerodynamic problem. They require to invert large sparse linear systems, which is done here using the sparse LU solver MUMPS ${ }^{63}$. In view of reproducibility, we make available at https: //bitbucket.org/jmoulin/pof2021 the codes that enable to solve the steady-state problem (7) and eigenproblem (8). We finally mention that our implementations have been validated with respect to the well-documented case of a heaving-spring-mounted circular cylinder undergoing vortex-induced vibrations in laminar flows. The results of this validation are reported in appendix B. 


\section{FLOW-INDUCED INSTABILITIES AT ZERO ANGLE OF ATTACK}

In this section, we investigate the various flowinduced instabilities that may emerge for spring-mounted plates immersed in viscous flows when varying the nondimensional parameters at zero steady angle of attack, $\alpha^{0}=0$. First, results of the fluid-solid stability analysis are described in section III A for fixed values of all nondimensional parameters, allowing a detailed description of the leading (i.e most unstable) modes: two coupledmode flutter (flutter and anti-flutter modes) and one vortex-shedding mode. Secondly, the effect of the reduced velocity $U^{*}$ is investigated in section III B where the transition from a vortex-induced vibration instability to coupled-mode flutter and finally to a steady divergence mode is analyzed. We finally investigate in section IIID the effect of the Reynolds number on coupled-mode flutter and divergence. For coupled-mode flutter in particular, we compare our approach to the classical Theodorsen model in order to show the magnitude of the viscous effects on this instability.

\section{A. Coupled-mode flutter and vortex-shedding instabilities}

We first investigate the case of a plate immersed in a flow at Reynolds number $\mathcal{R} e=2900$ and without angle of incidence, i.e. $\alpha^{0}=0$. The streamwise velocity (along $\mathbf{e}_{\mathbf{x}}$ ) of the steady solution is displayed in fig. 3(a) using the absolute velocity. As explained in the description of the fluid model (2), it is equal to zero away from the plate and to -1 , the (non-dimensional) velocity of the plate, at the wall. This solution is clearly symmetric with respect to the axis $x=0$ so that the steady lift and moment coefficients exerted on the plate are both equal to zero. For that low Reynolds number, the steady flow is mostly attached to the plate and, in particular, we do not observe any flow separation around the leading edge. A recirculation region, delimited by the black curve in the figure, is visible at the blunt trailing edge. Further downstream, the velocity profile is typical of a wake flow, with a velocity defect that decreases when progressing away from the plate.

The fluid-solid linear stability of that steady solution is then analyzed by solving the eigenproblem eq. (8) for the mass ratio $m=1000$ and an arbitrary reduced velocity of $U^{*}=4.7$, which has been chosen just high enough to exhibit a coupled-mode flutter instability, as we will see. The leading eigenvalues, i.e. the eigenvalues of largest growth rate, are displayed in fig. 3(b) in the complex plane $(\lambda, \omega)$, where $\lambda$ is the growth rate and $\omega$ the frequency. Note that only the eigenvalues of positive frequencies are shown here, since the eigenvalue spectrum is symmetric with respect to the real axis. Thus, all complex eigenvalues $(\omega \neq 0)$ appear in fact by pairs of complex conjugates. Most of the eigenvalues, marked with black crosses in the figure, are strongly stable $(\lambda<0)$.
On the contrary, the three eigenvalues, highlighted with colored circles, lie close to the neutral axis $\lambda=0$. As seen in the close-up view of the figure, one of them is unstable (red circle) while the other two (black and blue) are stable. In the following, we discuss the eigenmodes associated to these three eigenvalues.

The frequency of the unstable mode (red dot) $\omega=0.17$ is close to the natural heaving frequency of the plate $\omega_{0, h}=\Omega / U^{*}=0.8 / 4.7=0.17$. Its spatial structure is shown in fig. 4(a). The instantaneous pressure perturbation is depicted in the left figure with the blue-white colormap. The orange arrows correspond to the instantaneous displacement field of the rigid plate. Both are shown for the phase $\phi=\omega t=3 \pi / 4$ of the temporal oscillation. In the right part of the figure, we represent the oscillating part (i.e. neglecting the exponential growth) of the perturbation generated by the mode for the heaving $h^{1}(t)$ (solid line) and pitching $\theta^{1}(t)$ (dashed line) components. For the latter, we display the angle of attack that is opposite to the pitching angle, i.e. $-\theta^{1}(t)$. The pressure flow field exhibits extreme values close to the leading edge. The displacement field of the plate (orange arrows) couples the heaving and pitching motions. For that specific phase $\omega t=3 \pi / 4$, the angle of attack is close to its minimal values while the heaving displacement is close to its maximal value, as seen in the right figure. The phase shift between the heaving and pitching motion is close to $\pi$. By comparing the minimums of the heaving and pitching curves, we observe that the pitching signal (dashed line) leads on the heaving signal (solid line). This feature corresponds to a swimming-like motion of the airfoil that is typical of the coupled-mode flutter instability, as detailed in $\S 6.6$ of the book by Bisplinghoff and Ashley ${ }^{64}$ or in $\S 3.3 .2$ of the book by De Langre $^{65}$.

Let us now examine the stable mode depicted with the black dot in fig. 3(b). Its frequency $(\omega=0.15)$ is also close to the natural heaving $\left(\omega_{0, h}=\Omega / U^{*}=0.17\right)$ and pitching $\left(\omega_{0, \theta}=1 / U^{*}=0.21\right)$ frequencies. The corresponding eigenmode shown in fig. 4(b) exhibits very similar features to the flutter mode described above. However, the pressure field is of opposite sign for the same phase $\omega t=3 \pi / 4$, as the angle of attack is now close to its maximal value for that phase (see right figure). The displacement field still couples the heaving and pitching components but the latter is of larger amplitude, compared to the flutter mode. More interestingly, the phase shift between these two components is now close to $\pi / 2$ and the pitching motion (dashed curve) lags behind the heaving motion (solid). This mode is hereafter referred to as anti-flutter mode, as opposed to the unstable flutter mode described before. It should be noted here that both the flutter and anti-flutter modes can be obtained using simpler flow models such as a quasi-steady approach ${ }^{65}$, though the second is rarely discussed because it is stable.

The flutter and anti-flutter modes described above are only obtained when considering a fluid-solid stability analysis, unlike all the other modes in the eigenvalue 
(a)
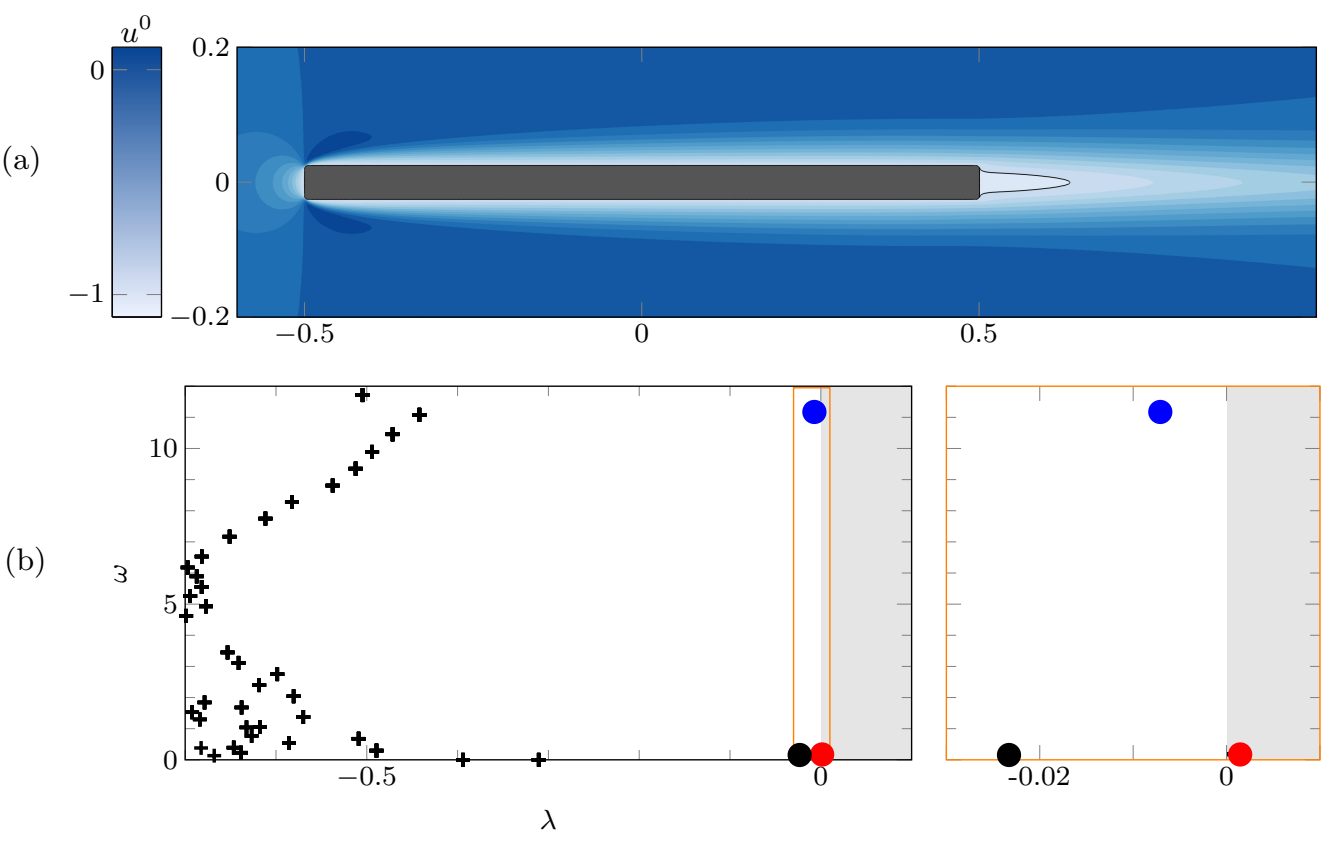

FIG. 3. Linear stability analysis of the spring-mounted plate at $U^{*}=4.7, \mathcal{R} e=2900$ and $m=1000$. (a) Streamwise velocity field of the steady solution and (b) eigenvalue spectrum of the fluid-solid stability problem with (right) a close-up view around the three modes of largest real part, highlighted with colored circles. The eigenvalues corresponding to the coupled-mode flutter instability are highlighted with red (flutter) and black (anti-flutter) dots while the eigenvalue corresponding to the wake instability is marked with the blue dot. The spatial structure of the corresponding eigenmodes is depicted in fig. 4 .

spectrum of fig. 3(b) that can also be obtained with a purely-hydrodynamic stability analysis. The latter consists in computing the leading eigenvalues of the fluid Jacobian matrix $\mathscr{J}_{\mathrm{ff}}$. Most of these eigenvalues are strongly damped, but for the one highlighted with the blue dot. Its negative growth rate is very close to zero and its frequency $(\omega=11.2)$ is much higher than the natural heaving and pitching frequencies. The spatial structure of this high-frequency mode, shown in fig. 4(c), exhibits very different features compared to the flutter and anti-flutter modes. The solid perturbation (heaving and pitching) seen in the right figure is negligible because the flow perturbation associated to this mode does not induce significant lift or moment on the plate. Indeed, the pressure fluctuation is negligible close to the plate and large amplitude fluctuations are only observed in its wake, where the spatial pattern oscillates in the stream-wise direction. This is a typical feature of vortex-shedding modes that get unstable in the wake of bluff bodies ${ }^{66-68}$ where the steady solution exhibits a recirculation region. In the case of the fixed plate, the purely-hydrodynamic mode gets unstable around $\mathcal{R} e_{c \text {,wake }} \simeq 2925$ and its Strouhal number based on the thickness $\tilde{e}$ of the plate is $\mathcal{S}_{\tilde{e}}=(\tilde{e} / \tilde{c}) \omega /(2 \pi) \simeq 0.09$. It is difficult to discuss precisely this value in regards of previous works ${ }^{69-71}$ since the latter often focused on higher Reynolds numbers where nonlinear effects involving both leading and trailing edge vortex shedding come into play.

\section{B. Effect of the reduced velocity}

Keeping the mass ratio to $m=1000$, we now vary the reduced velocity in the range $0.05 \leq U^{*} \leq 8$ (or similarly the natural pitching frequency in the range $20 \geq \omega_{\theta}^{0} \geq$ $0.125)$ and investigate the effect on the three eigenvalues identified in the previous section. Figure 5 shows the path of these eigenvalues in the complex plane. The case $U^{*}=4.7$ explored in the previous section is marked with dots of colors identical to fig. 3(b), while small circles correspond to the eigenvalues obtained for other values of $U^{*}$, with a varying step size. The right figures are closeup views around high (top) and low (bottom) frequencies. The dashed lines materialize the paths of the natural heaving (red) and pitching (black) modes.

Let us first examine the path of the red eigenvalue that corresponds to the flutter mode at $U^{*}=4.7$. When increasing the reduced velocity further above 4.7 , this unstable flutter mode tends to restabilize. We will see later in fig. 8 that it is even stable again for very high $U^{*}$. When decreasing $U^{*}$, the flutter mode stabilizes at $U^{*}=4.5$. Its path (red solid curve) then closely follows the locus of the neutrally stable $(\lambda=0)$ natural heaving mode (red dashed line). The light damping of the red branch in comparison to the natural heaving mode is due to the interaction with the flow. However, when the frequency of the heaving mode gets close to the frequency of the vortex-shedding mode (blue) for small $U^{*}$, the modes interact leading to a destabiliza- 

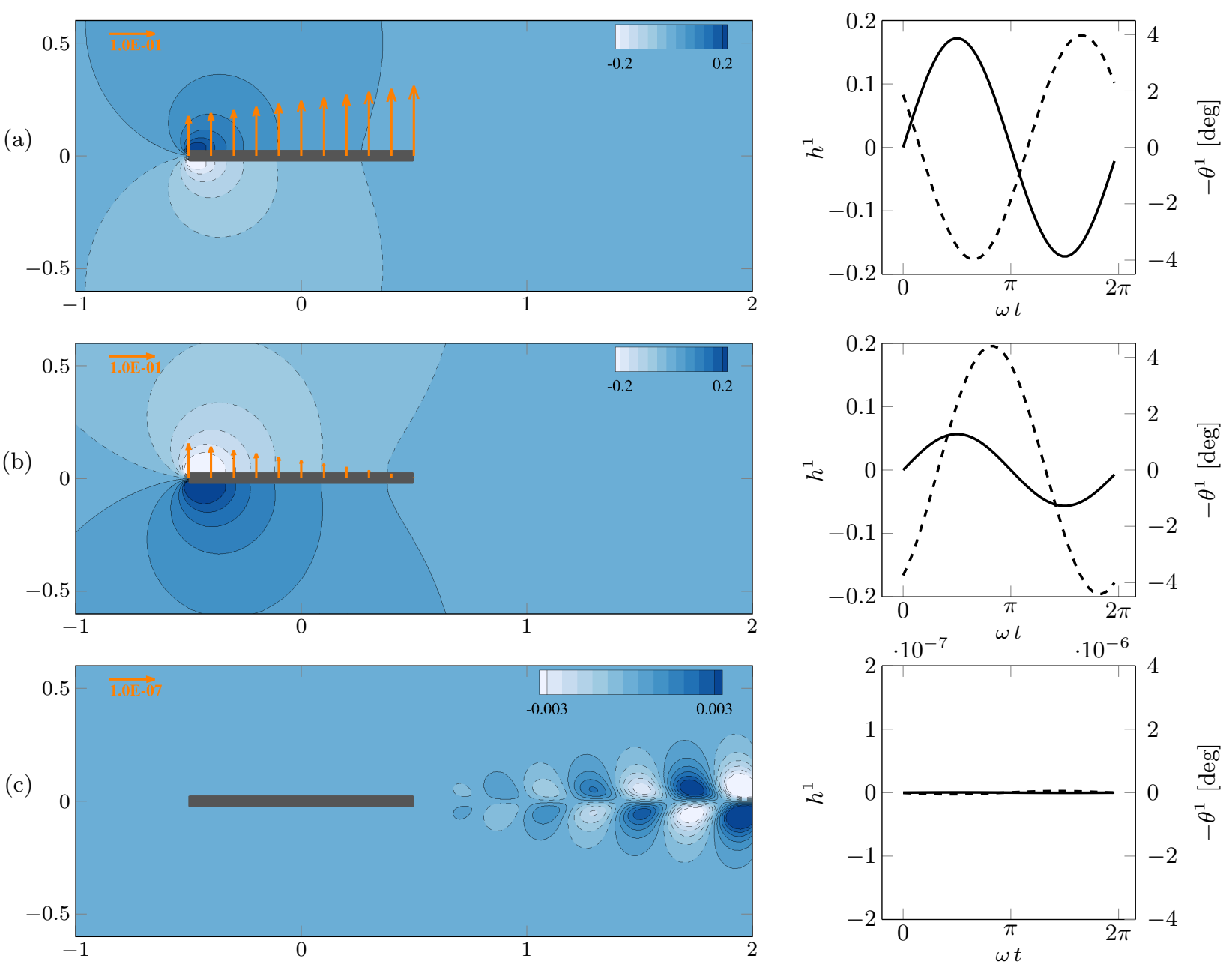

FIG. 4. Eigenmodes corresponding to the three eigenvalues of fig. 3(b) marked with colored dots: (a) red, unstable flutter mode; (b) black, stable anti-flutter mode; (c) blue, stable vortex-shedding mode. The left figures display a snapshot of the eigenmode at phase $\omega t=3 \pi / 4$ of the oscillation. The colormap depicts the pressure flow field, while the vectors represent the displacement field of the plate. The right figures show the vertical displacement (solid line) and instantaneous angle of attack (dashed line) as a function of the phase $\omega t$.

tion of the red eigenvalue between $U_{\mathrm{V} 1}^{*}=0.0712$ and $U_{\mathrm{V} 2}^{*}=0.0725$, as emphasized in the close-up view (highfrequency). The interaction between these two modes is revealed by the shape of the eigenmode corresponding to the red eigenvalue. The pressure field, shown in fig. 6(a), is very similar to that of the vortex-shedding mode, already shown in fig. 4(c). In particular, the lift force exerted on the plate is small. However, the perturbation of the plate motion is significantly different since the heaving component is not negligible anymore (unlike the pitching component that remains negligible). This is characteristic of a resonance-like phenomena where a small lift force may induce a large heaving displacement. A similar resonance effect has been reported and described on spring-mounted circular cylinder flows, based on stability analysis ${ }^{38,40,72}$ and nonlinear temporal simulations $^{35,39,40}$. More recently similar phenomena were highlighted for spring-mounted airfoils undergoing tran- sonic buffet flow conditions ${ }^{42,43}$.

Let us now examine the black eigenvalue whose path in the complex plane closely follows that of the natural pitching mode (black dashed line) when it is stable at low reduced velocity. As $U^{*}$ increases, the growth rate increases and the frequency $\omega$ suddenly deviates from the natural pitching frequency to reach zero. Then it gets unstable as shown in the close-up view (low frequency) in fig. 5. The mode corresponding to this eigenvalue is presented in fig. 6(b). This destabilization of the pitching mode by a static instability $(\omega=0)$ is an example of the classical divergence instability ${ }^{1}$. Note that here, the divergence mode includes both heaving and pitching displacements. However, it is well-known that divergence is possible with only the pitching degree of freedom, for example in the thin airfoil theory limit.

To better analyze the flutter and divergence instabilities, we display in fig. 7 the evolution of their growth 

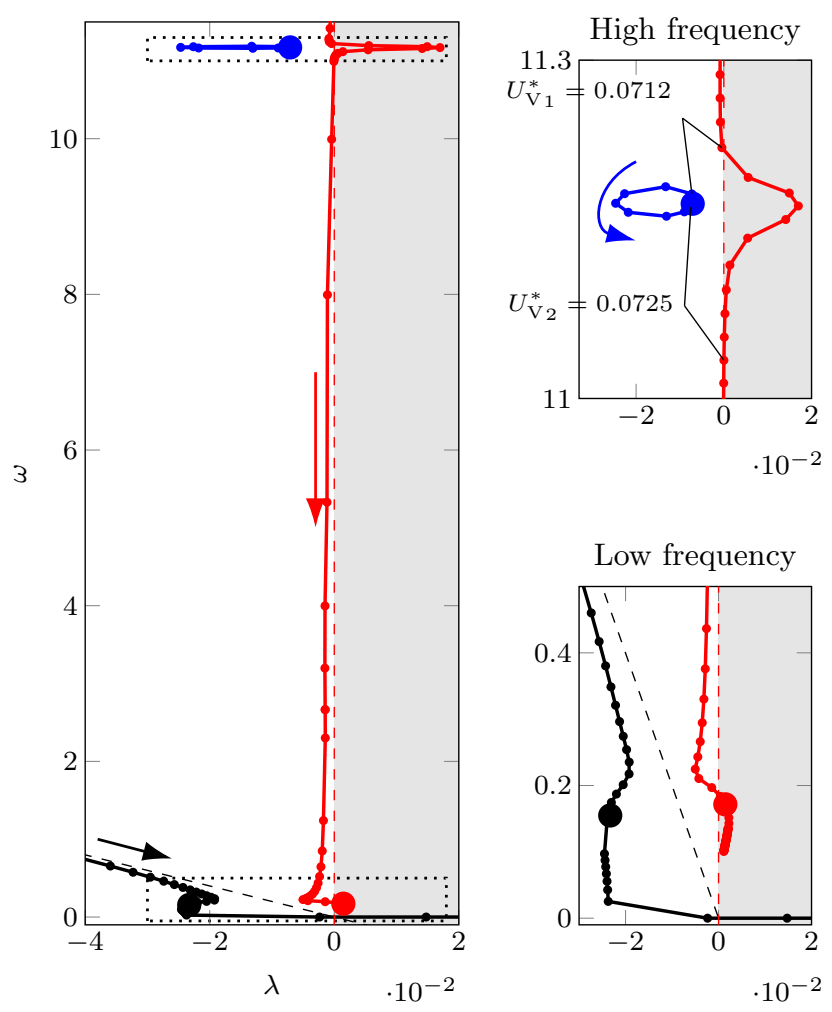

FIG. 5. Paths of the three eigenvalues in the complex plane when varying the reduced velocity in the range $0.05 \leq U^{*} \leq$ 8. The big colored dots correspond to the eigenvalues at $U^{*}=4.7$ previously shown in fig. $3(\mathrm{~b})$. The dashed straight lines indicate the paths of the natural solid modes (heaving in red, pitching in black). The dotted rectangles materialize the zones of VIV type and flutter/divergence type instabilities, of which magnified views are exposed in the right part of the figure. The filled gray area represents the unstable half plane.

rate (top) and frequency (bottom) as a function of the reduced velocity $U^{*}$, which is here varied in the range $0.5 \leq$ $U^{*} \leq 8$. The lower bound being larger than the range for vortex-induced vibrations $0.0712 \leq U^{*} \leq 0.0725$ (top right part of fig. 5), the latter are not observed. The growth rate $\lambda$ and frequency $\omega$ of the eigenvalues are both normalized by the natural pitching frequency $\omega_{0, \theta}$. With this new scaling, we avoid the large variation of frequency observed in fig. 5 , the latter remaining close to unity. It is clear in fig. 7 that for $U^{*} \rightarrow 0$, the red and black modes respectively tend towards the solid natural eigenmodes:

$$
\begin{aligned}
& \frac{\lambda_{h}+\mathbf{i} \omega_{h}}{\omega_{0, \theta}}=\left(-\Omega \zeta_{h}+\mathbf{i} \Omega \sqrt{1-\zeta_{h}^{2}}\right)=0+0.8 \times \mathbf{i} \\
& \frac{\lambda_{\theta}+\mathbf{i} \omega_{\theta}}{\omega_{0, \theta}}=\left(-\zeta_{\theta}+\mathbf{i} \sqrt{1-\zeta_{\theta}^{2}}\right)=-0.05+0.999 \times \mathbf{i}
\end{aligned}
$$

As $U^{*}$ increases, the frequencies of the branches first approach each other while growth rates slightly decrease. Around $U^{*}=4$, both branches have equal frequency and a brutal increase of the growth rate of the heaving branch is observed so that the heaving branch goes unstable at the critical flutter velocity, $U_{F}^{*}=4.5$. As a consequence, flutter is linked to the heaving and pitching frequencies approaching each other. For even higher $U^{*}$, the pitching branch finally gets unstable through the static divergence mode (fig. 6(b)), at a critical divergence velocity of $U_{\mathrm{D}}^{*}=7.4$. Let us finally mention that in the particular case studied here, it is the heaving branch that gets unstable via the flutter instability. However, by changing the damping parameters $\zeta_{h}$ and $\zeta_{\theta}$, we could observe different scenarios where it is the pitching branch that gives rise to flutter.

\section{Effect of the mass ratio}

In the previous sections, we identified the critical velocities $U_{\mathrm{D}}^{*}, U_{F}^{*}$ and $U_{\mathrm{V}}^{*}$ for the destabilization of the divergence, flutter and vortex-induced vibrations modes at Reynolds number $\mathcal{R} e=2900$ and mass $m=1000$. Keeping the Reynolds number fixed, we now vary the mass ratio and use the continuation method introduced in section II B to determine, in the parameter space $\left(m, U^{*}\right)$, the neutral curves corresponding to each instability.

The neutral curves $U_{\mathrm{D}}^{*}(m), U_{F}^{*}(m)$ and $U_{\mathrm{V}}^{*}(m)$ corresponding to the divergence, flutter and vortex-induced vibrations instabilities are shown in fig. 8 in a log-log plot with grey, red and blue curves, respectively. The instability regions being shaded with the corresponding colors, the white area is the stable region. In this figure, we do not display the frequency of the corresponding critical eigenmode, but we stress that its order of magnitude is that of the natural solid modes, represented on the right vertical axis by the pitching frequency $\omega_{0, \theta}=1 / U^{*}$. We clearly identify two regions of instabilities: (i) the highreduced-velocity region (upper part of the figure) where flutter and divergence occur with low or zero frequency and (ii) the low-reduced-velocity region (lower part of the figure) where the vortex-induced vibration instability occurs in a narrow range of high frequencies.

Let us first consider the neutral curves for flutter (red) and divergence (grey). At high mass ratio, the flutter instability occurs at critical reduced velocities smaller than for the divergence instability. When decreasing the mass ratio, the critical reduced velocity decreases in both cases, with a slope close to $1 / 2$ in the log-log plot. The neutral curve of the divergence instability is in fact a straight line that is in good agreement with the theoretical expression $U_{\mathrm{D}}^{*}(m)=\sqrt{r_{\theta}^{2} /(\pi / 2) m}$ obtained under the hypothesis of pure pitching motion and thin airfoil theory modelling the aerodynamics ${ }^{2}$. When decreasing the mass ratio, the slope of the flutter neutral curve tends to decrease, while the flutter frequency increases. For instance, the critical frequency is $\omega_{\mathrm{c}} \sim \mathcal{O}(0.1)$ for $m \sim 10^{3}$ and $\omega_{\mathrm{c}} \sim \mathcal{O}(1)$ for $m \sim 10$. As $m$ further decreases, the critical flutter velocity reaches its minimal value around $m \simeq 20$ before increasing and crossing the neutral curve for divergence instability at $m \simeq 11$. For 

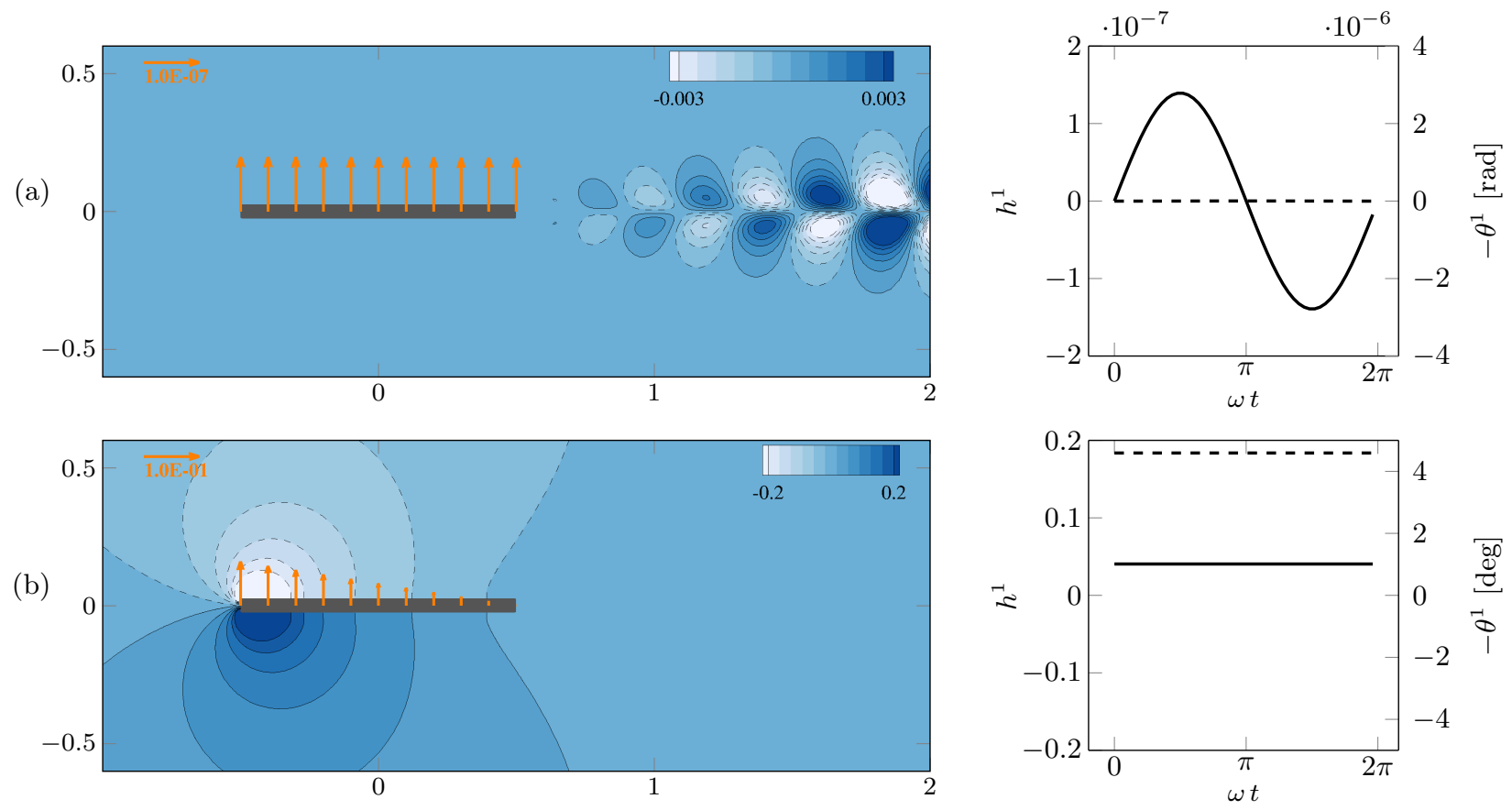

FIG. 6. Eigenmodes corresponding to (a) the (heaving) VIV instability $\left(U^{*}=0.0716\right)$ and (b) the divergence instability $\left(U_{\mathrm{D}}^{*}=7.4\right)$ observed in fig. 5. Color codes and legends are identical to fig. 4 .

lower mass ratio, the divergence instability thus occurs prior to the flutter instability that may not even occur for $m \leq 7$. Indeed, we observe a turning point in the neutral curve of flutter. As a consequence, for $m>7$, there exists a reduced velocity higher than $U_{F}^{*}$ for which the flutter mode restabilizes. To our best knowledge, this "second" neutral curve for which the flutter mode restabilizes at high reduced velocity is reported here for the first time. This effect and the underlying physical mechanism are further discussed in the next subsection when investigating the effect of viscosity. At the intersection of the flutter and divergence neutral curves, a codimensiontwo point can be identified for a particular mass ratio $m^{I I} \simeq 11$ and $U^{*} \simeq 0.9$. At this point, both the flutter and divergence modes are neutrally stable and could be involved in the nonlinear dynamics. Typically, the simultaneous presence of these two modes may explain the occurrence of so-called "dynamic divergence" 73,74 where a combination of oscillations and mean deviation of the wing/plate is observed in the nonlinear regime. These studies were performed at higher Reynolds numbers than ours $\left(\mathcal{R} e \simeq 10^{6}\right.$ ), on a different 2D section (NACA0016 airfoil) and with different structural parameters. However, the range of dynamic divergence reported there is roughly $m \in[1.6,6.3]$, which is the same order of magnitude as the values found in the present work.

Finally, we consider the region of vortex-inducedvibration instability depicted in blue in fig. 8. Whatever the mass ratio, this instability occurs at much lower values of $U^{*}$ compared to the flutter and divergence instabilities. Its typical frequency $\omega_{\mathrm{c}} \sim 10$ barely de- pends of the mass ratio, unlike the shape of the neutral curve. For high $m$, the vortex-induced vibration mode is unstable in a very narrow range of reduced velocity around $U^{*} \simeq 0.07$. It is associated to a resonance between the solid heaving mode and the vortex-shedding mode, that occurs when their frequency coincide, i.e. $\omega_{0, h}=\Omega / U^{*} \simeq \omega_{\text {wake }}$, as indicated in the figure with the lower dashed line. For intermediate $m$, a second region of instability appears for slightly larger reduced velocity $U^{*} \simeq 0.09$. It is then associated to the resonance of the vortex-shedding mode with the pitching mode when $\omega_{0, \theta}=1 / U^{*} \simeq \omega_{\text {wake }}$ (upper dashed line). The existence of two separated instability regions at intermediate mass ratio is thus clearly related to the resonance of the vortex-shedding mode with the two (heaving and pitching) solid modes at different $U^{*}$. When decreasing $m$, these two instability regions widen in $U^{*}$, as for the case of a spring-mounted cylinder ${ }^{40}$, until they merge around $m=8$. For lower mass ratio, vortex-induced vibrations may thus occur far enough from the resonance conditions marked by the thin horizontal dashed lines.

\section{Effect of viscosity}

In the previous sections we explored the linear behavior of the spring-mounted plate by varying some classical aeroelastic parameters that are the reduced velocity and the mass ratio. We now exploit the full potential of a global stability approach by studying how viscosity affects flutter and divergence. Its effect on the VIV in- 

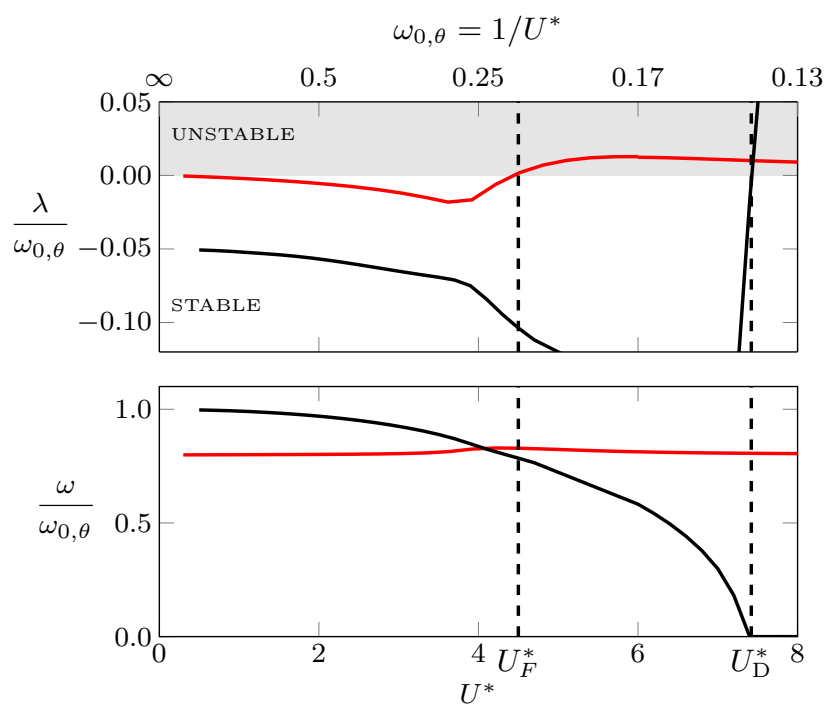

FIG. 7. Evolution of the flutter and anti-flutter eigenmodes, marked respectively by the red and black disks in fig. 3(b), as a function of the reduced velocity $(\mathcal{R} e=2900, m=1000)$. The flutter critical velocity $U_{F}^{*}$ is defined as the reduced velocity for which the flutter mode represented in fig. 4(a) becomes unstable. For even higher $U^{*}$, a static divergence instability occurs for $U^{*}>U_{\mathrm{D}}^{*}$. As $U^{*} \rightarrow 0$ both branches tend towards the solid-only eigenmodes. For the particular parameters used in the figure, those modes reduce to $\sigma=\mathbf{i} 0.8 \omega_{0, \theta}$ (heaving mode) and $\sigma=(-0.05+\mathbf{i} 0.999) \omega_{0, \theta}$ (pitching mode).

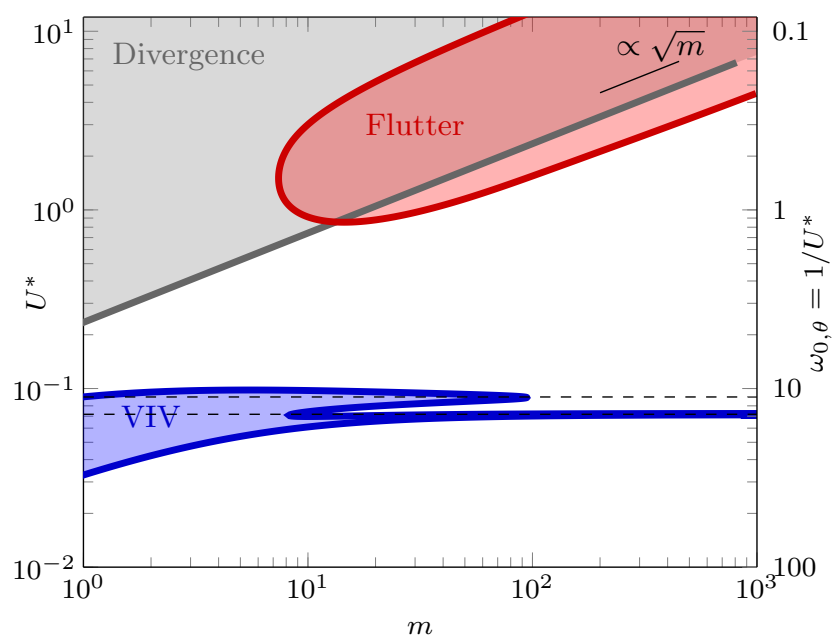

FIG. 8. Neutral curves and instability regions for the flutter (red), divergence (gray) and vortex-induced-vibration (blue) modes in the parameter space $\left(m, U^{*}\right)$ or equivalently $\left(m, \omega_{0, \theta}\right)$. The two horizontal dashed lines indicate the value of the reduced velocity for which the natural heaving (lower line) and pitching (upper line) frequencies correspond exactly to the frequency of the vortex-shedding mode $(\mathcal{R} e=2900$, $\alpha^{0}=0^{\circ}$.
TABLE II. Effect of the Reynolds number on the steady lift and moment slopes at $\alpha^{0}=0$. The last column recalls the potential flow values from thin airfoil theory.

\begin{tabular}{lccccc}
\hline \hline $\mathcal{R} e$ & 10 & 110 & 500 & 2900 & $\infty$ \\
\hline$\frac{\mathrm{d} \mathcal{C}_{L}^{\text {stat }}}{\mathrm{d}(-\theta)}$ & 3.22 & 4.07 & 4.88 & 5.88 & $2 \pi$ \\
$\frac{\mathrm{d}\left(-\mathcal{C}_{M}^{\text {stat }}\right)}{\mathrm{d}(-\theta)}$ & 0.89 & 1.04 & 1.23 & 1.52 & $\pi / 2$ \\
\hline \hline
\end{tabular}

stability is left aside and we refer the interested reader to dedicated works ${ }^{40,72}$.

\section{Flutter and divergence thresholds for different Reynolds numbers}

In fig. 9, we show the flutter (red) and divergence (gray) neutral curves for three different Reynolds numbers spanning three orders of magnitude: $\mathcal{R} e=10(\mathrm{a})$, $\mathcal{R} e=110$ (b) and $\mathcal{R} e=2900$ (c). First, by looking for example at the value of $U_{\mathrm{D}}^{*}$ at $m=1$, one can observe that the divergence curve is shifted towards lower $U^{*}$ as $\mathcal{R} e$ increases. This trend is linked to the increase in the slope of the steady lift $\mathcal{C}_{L}^{\text {stat }}$ and moment $\mathcal{C}_{M}^{\text {stat }}$ coefficients as $\mathcal{R} e$ increases (see table II), which naturally leads to lower divergence velocities according to the classical formula for torsional divergence: $U_{\mathrm{D}}^{*}(m)=\sqrt{r_{\theta^{2}} /\left(\mathrm{d} \mathcal{C}_{M}^{\text {stat }} /\left.\mathrm{d} \theta\right|_{\theta^{0}}\right) m}$. For the flutter threshold, a similar trend is observed in the high- $m$ limit. For lower mass ratios, we note a large change in the position of the folding point point that drops from $m \sim 300$ to $m \sim 20$ when increasing the Reynolds number from $\mathcal{R} e=10$ to $\mathcal{R} e=110$. Between $\mathcal{R} e=110$ and the baseline case at $\mathcal{R} e=2900$, further shift is observed but in less important proportions. In addition, it is observed that the high- $U^{*}$ boundary of the flutter curve varies significantly more with $\mathcal{R} e$ than the low- $U^{*}$ boundary. It indicates that the high- $U^{*}$ restabilization of the flutter mode is strongly dependent on viscous effects and tends to explain why it was never reported before, in high- $\mathcal{R} e$ flows. More insights on that aspect are provided in the next paragraph where we compare the present approach with the classical Theodorsen theory.

Due to the wide variations of the high- $U^{*}$ limit of the flutter region, two scenarios are possible depending on $\mathcal{R} e$. At high-enough $\mathcal{R} e$ (c), a classical scenario is observed where, as one increases $U^{*}$, the flutter mode destabilizes first, remains unstable and then the divergence mode destabilizes. On the contrary, at low Reynolds (a), the flutter mode destabilizes first, then restabilizes - leaving the system stable on some narrow range of $U^{*}$ - and finally divergence is triggered. 


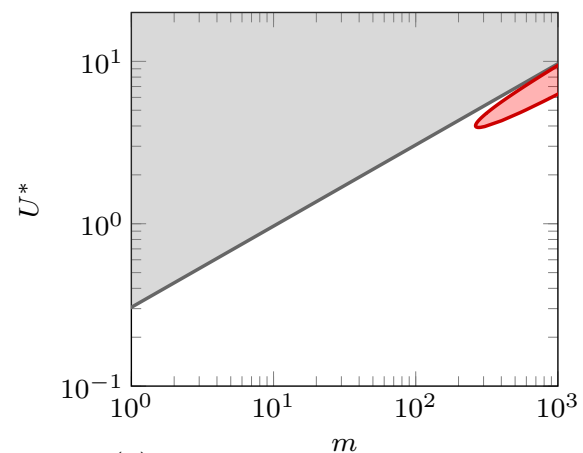

(a)

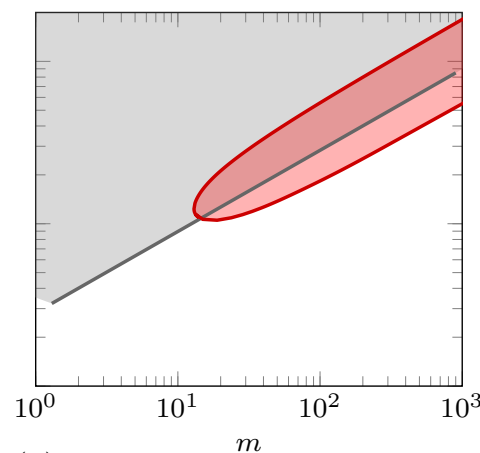

(b)

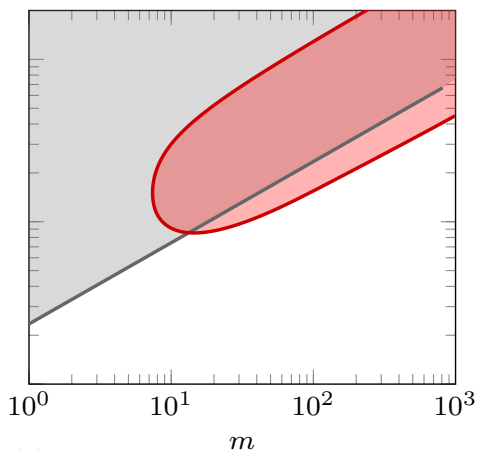

(c)

FIG. 9. Linear stability neutral curves for flutter (red) and divergence (gray) instabilities in the plane $\left(m, U^{*}\right)$ for $(\mathrm{a}) \mathcal{R} e=10$, (b) $\mathcal{R} e=110$ and (c) $\mathcal{R} e=2900$. The unstable regions are shaded as in fig. 8 .

\section{A comparison of viscous flutter thresholds to the Theodorsen model}

In this section, we compare the flutter stability results from the global stability approach with the ones obtained using a statically calibrated version of the Theodorsen ${ }^{27}$ theory. Indeed, if the original Theodorsen model makes the hypothesis of potential flows, it is of common use ${ }^{14}$ to empirically extend its range of application to viscous flows by calibrating it using the steady viscous lift and moment slopes from table II instead of the original values from thin airfoil theory. In the following, we will use this simple-enough calibration strategy as we believe it offers a fairer comparison of the present computational approach with an analytical Theodorsen(-like) approach. Details of this static calibration procedure are reported in appendix $\mathrm{C}$.

The flutter neutral curves obtained with the global stability approach (solid) and the calibrated Theodorsen model (dashed) are reported in fig. 10(a) for the three Reynolds numbers $\mathcal{R} e=10$ (brown), $\mathcal{R} e=100$ (gray) and $\mathcal{R} e=2900$ (black). First, in the limit of high $m$, the Theodorsen curves tend towards the viscous curves. This is shown more quantitatively in fig. 10(b) where the relative error between both approaches for the low- $U^{*}$ flutter boundary is presented. Focusing temporarily on the case $\mathcal{R} e=2900$ (black), we observe that the Theodorsen model remains fairly close (less than $10 \%$ deviation) to the Navier-Stokes prediction as the mass ratio decreases down to $m \simeq 10^{2}$. For lower values, the critical velocity is increasingly overestimated by Theodorsen, of as much as $35 \%$ at $m=10$. For $\mathcal{R} e=110$, similar observations can be made. For the lowest Reynolds, $\mathcal{R} e=10$ (brown), the Theodorsen model fails in predicting the rapid shift of the turning point to higher mass ratios. As a consequence, it predicts the occurence of flutter for mass ratios $10 \leq m \leq 200$ where it cannot appear according to global stability.

For all three Reynolds numbers, the Theodorsen model predicts a vertical asymptote of the neutral curve for mass ratios between 5 and 10 . This is clearly different from the global stability results that indicate a turning point and subsequent high- $U^{*}$ restabilization of the flutter mode, as described in the previous section. Some intuition about this observation may be gained by thinking of the Theodorsen model as an asymptotic limit of the global stability approach for large Reynolds numbers. Indeed, if the high- $U^{*}$ flutter restabilization tends to delay as $\mathcal{R} e$ increases, then it is expected to never occur for $\mathcal{R} e \rightarrow \infty$. This corresponds to the asymptotic behaviour of the Theodorsen model. Overall, the aforementioned discrepancies between the statically calibrated Theodorsen model and the global stability results suggest that significant unsteady viscous effects - that cannot be captured with a static calibration of a potential model - come into play at the Reynolds numbers considered here.

\section{EFFECT OF THE STEADY ANGLE OF ATTACK}

We end this study by investigating the effect of the steady angle of attack $\alpha^{0}$ of the plate on the flutter instability. As this parameter modifies the steady solutions around which the fluid-solid perturbations develop, we first describe the evolution of this steady solution. Results are displayed in fig. 11 for the Reynolds number $\mathcal{R} e=500$. For low angles of attack, the lift and moment coefficients shown in fig. 11(a) evolve linearly. The steady flow, depicted in fig. 11(b) with the streamwise velocity, is attached to the plate until $\alpha^{0}=6^{\circ}$ (except for the small recirculation region visible at the trailing edge). When increasing the angle of attack in $6^{\circ}<\alpha^{0}<10^{\circ}$, a flow separation first occurs at the trailing edge and then progressively moves towards the leading edge. The slope of the lift and moment coefficients then diminishes. When they reach their maximal values slightly below $\alpha^{0}=10^{\circ}$, the flow is fully separated, as seen in fig. 11(b). The recirculation region in the wake of the plate keeps growing when further increasing the angle, leading to a slight decrease of the magnitude of the lift and moment coefficients. This behaviour is similar 

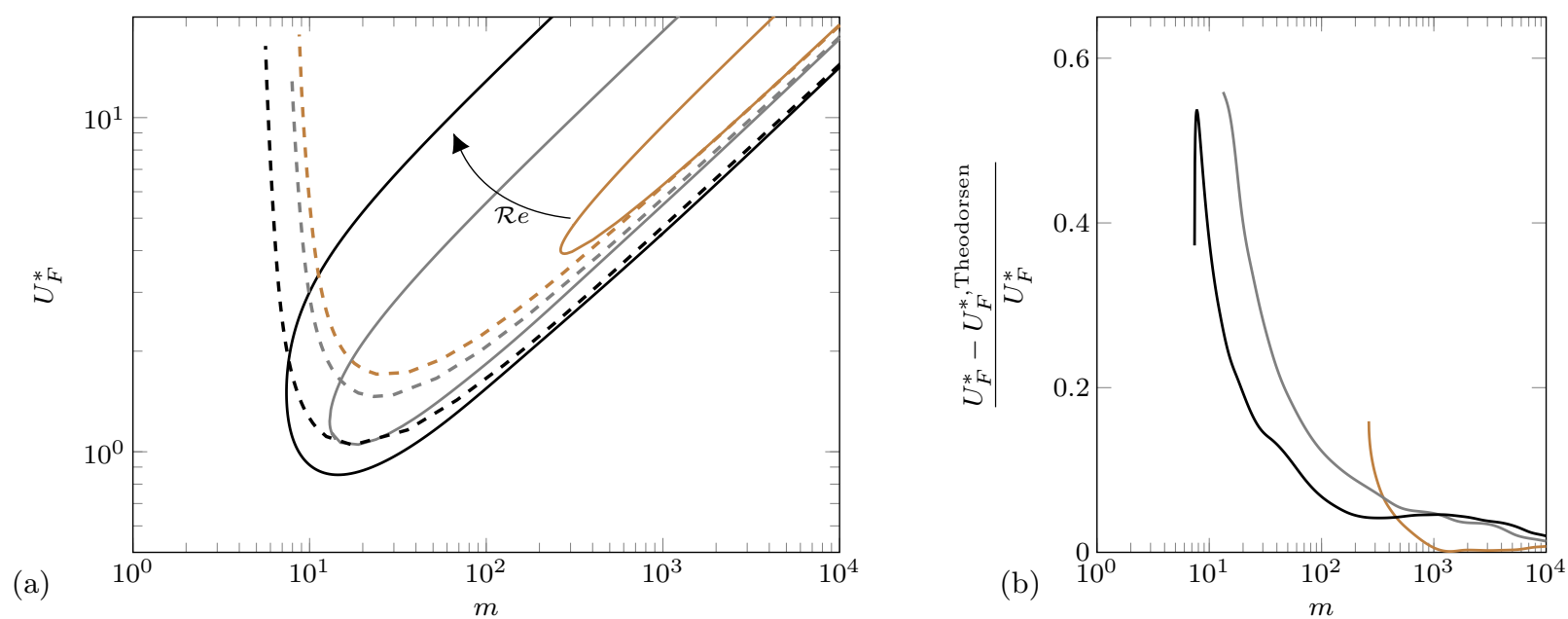

FIG. 10. (a) Comparison of the statically calibrated Theodorsen model (dashed) and the global stability approach (solid) for the prediction of flutter neutral curves at different Reynolds numbers: $\mathcal{R} e=10$ (brown), $\mathcal{R} e=110$ (gray), $\mathcal{R} e=2900$ (black). For a clear identification of the stable/unstable regions, the reader is reported to fig. 8. (b) Relative error for the prediction of the low- $U^{*}$ flutter threshold between the global stability and calibrated Theodorsen approaches.

to the stall of airfoils that occurs for turbulent flows at higher Reynolds number ${ }^{3,4}$, but the lift drop is much less pronounced. Such a light stall is a known feature of lowto-moderate Reynolds flows around plates ${ }^{14}$ or airfoils ${ }^{75}$. A purely-hydrodynamic stability analysis of these steady solutions reveals the destabilization of a unsteady mode for $\alpha^{0} \geq 9.8^{\circ}$. This is a vortex-shedding mode whose frequency $\omega_{\text {wake }} \simeq 4$ can be rescaled with the frontal length $\sin \left(\alpha^{0}\right)$ yielding the Strouhal number $S t=0.68$. The steady flow is thus unstable for $\alpha^{0} \geq 9.8^{\circ}$, as emphasized with the dashed line in fig. 11(a). The steady lift and moment coefficients reported for such high angles should then be interpreted with caution, when comparing with time-averaged results of temporal simulations or experiments. Indeed, the flow unsteadiness is likely to modify the steady flow, so that the mean lift value progressively drift away from the steady lift reported here. To avoid such effects, the subsequent discussion on the flutter instability is restricted to $0^{\circ} \leq \alpha^{0} \leq 10^{\circ}$.

In fig. 12(a), we present the evolution of the heaving (red curves) and pitching (black curves) eigenvalues as a function of the reduced velocity for different values of the steady angle of attack. The case $\alpha^{0}=0^{\circ}$ (dashed curves) corresponds to the typical coupled-mode flutter (CMF) scenario already described in section III B. When increasing the reduced velocity, the frequencies of the two modes (bottom figure) approach each other leading to the destabilization of the heaving mode (red dashed curve in top figure). When increasing the angle up to $\alpha^{0}=6^{\circ}$ (dash-dotted curve), a coupled-mode flutter scenario is also observed but with slight differences. Indeed, the frequency of the two modes gets close to each other for higher reduced velocity, thus delaying the occurence of flutter. Moreover, increasing the reduced velocity tends to stabilize the heaving mode (red) and to destabilize the pitching mode (black). Thus, it is the pitching mode, rather than the heaving mode, that gets unstable for that angle. Therefore, as the angle increases in $0^{\circ} \leq \alpha^{0} \leq 6^{\circ}$, there is a smooth transition from a heaving coupled-mode flutter to a pitching coupled-mode flutter. The critical reduced velocity $U_{F}^{*}$, shown in fig. 12(b), progressively increases with the angle of attack.

For $\alpha^{0} \geq 7$, the coupled-mode flutter scenario is not observed anymore. The destabilization of the pitching mode (black dotted curve, $\alpha^{0}=8^{\circ}$ ) still occurs although the heaving and pitching frequencies remain well separated. This is referred to as a pitching Single-Mode Flutter scenario (pSMF). Instead of a coupled heaving an pitching motion, the critical mode for pSMF mainly oscillates along the pitching degree of freedom, as illustrated in fig. 13(a). As seen in fig. 12(b), a strong decrease of the critical reduced velocity is observed after the transition from CMF to pSMF around $\alpha^{0} \sim 7^{\circ}$. It is interesting to note that the features reported above have been observed experimentally, for example by Razak, Andrianne, and Dimitriadis ${ }^{6}$, where the flutter instability on a heaving and pitching NACA0012 profile was shown to transition from CMF to pSMF as the static angle of attack is increased. However, the higher Reynolds number $\left(\mathcal{R} e \simeq 10^{5}\right)$ and different airfoil profile used in their study does not allow a quantitative comparison with our results.

For $\alpha^{0}=10^{\circ}$, we notice in fig. 12(a) that, in addition to the pitching mode (solid black line) becoming unstable around $U_{F}^{* \text { pSMF }}=4.8$, the heaving mode (solid red line) is also very close to unstable. It actually does become slightly unstable around $U_{F}^{* \mathrm{hSMF}}=5.2$, giving rise to a secondary heaving Single-Mode Flutter (hSMF), where the eigenmode mainly oscillates along the heaving degree of freedom (see fig. 13(b)). The frequency at criticity is close to the one of the natural heaving mode, i.e. $\omega_{\mathrm{c}}^{\mathrm{hSMF}}=\Omega / U_{F}^{* \mathrm{hSMF}} \sim 0.15$. This low-frequency indi- 

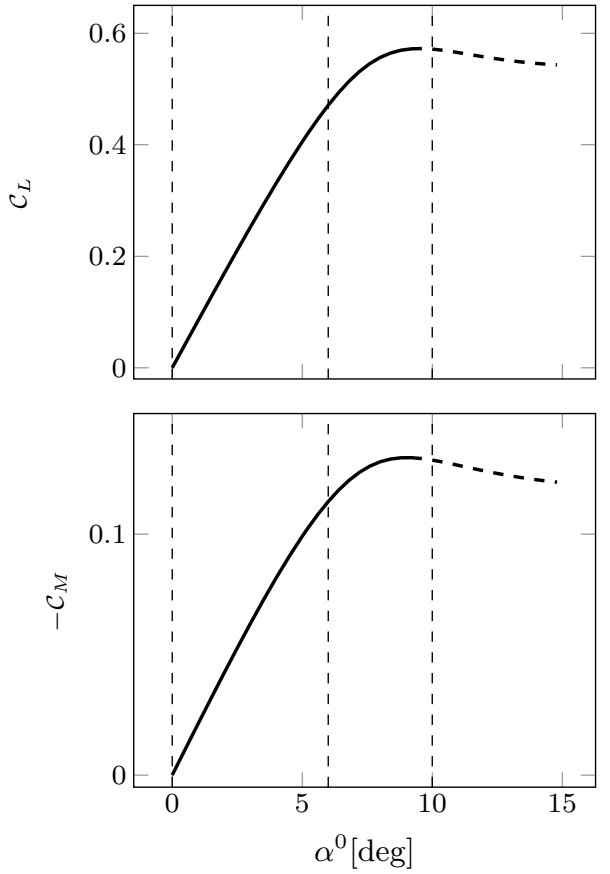

(a)
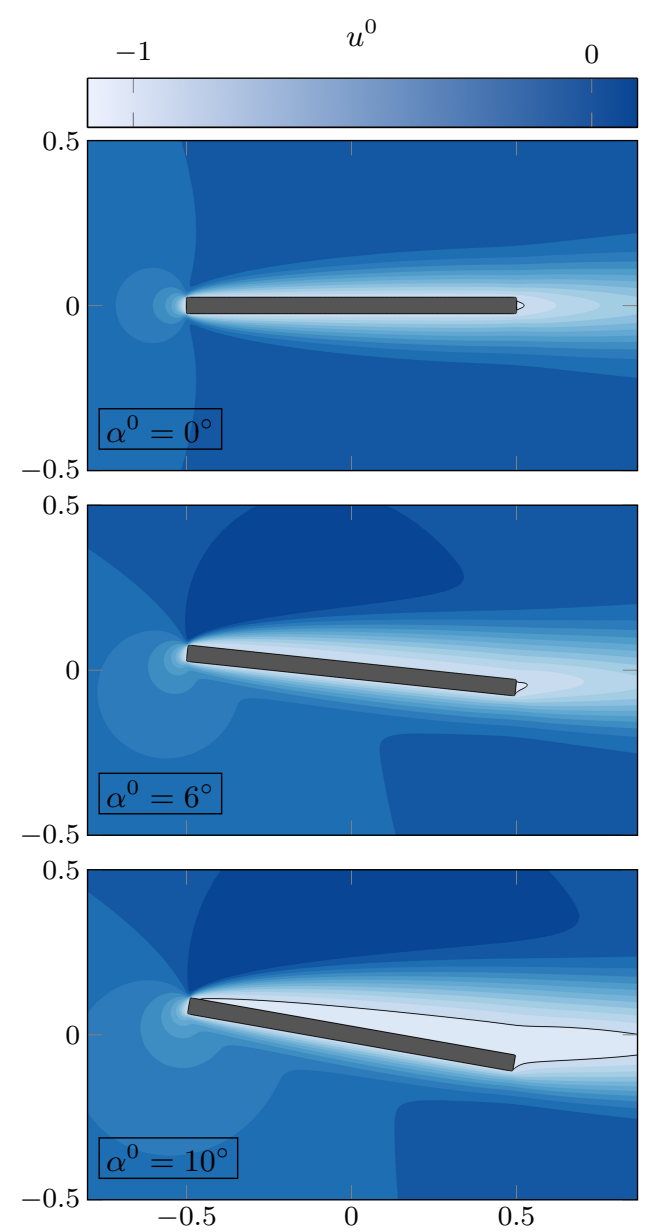

(b)

FIG. 11. Characteristics of the steady flow around the fixed plate at $\mathcal{R} e=500$ and for different angles of attack. (a) Aerodynamic coefficients as a function of the angle of attack $\alpha^{0}$. Stable and unstable solutions are shown with solid and dashed curves, respectively. (b) Streamwise velocity field shown for different angles of attack indicated in the figures and reported in (a) with vertical dashed lines. Black curves delimit the regions of flow recirculation.

cates that hSMF is another aeroelastic instability, wellseparated from the high-frequency wake-mode $\left(\omega_{\text {wake }}=4\right.$ at $\left.\alpha^{0}=10^{\circ}\right)$. The critical velocity for hSMF is materialized in fig. 12(b) by the red disk. We should mention that hSMF could have been anticipated already by observing the negative slope of the lift curve fig. 11(a) at $\alpha^{0}=10^{\circ}$. Indeed, using quasi-steady arguments, it is well-known that a negative steady lift slope may induce negative aerodynamic damping on an only-heaving body, provided the Glauert-Den Hartog criterion is met ${ }^{32}$, which we verified (not shown) it is here for $\alpha^{0}=10^{\circ}$.

\section{CONCLUSION}

In this paper, we revisited the linear stability of a typical aeroelastic section with an incompressible NavierStokes flow model. The linear stability of the fluid-solid system was assessed using a global stability approach which consists in scrutinizing the eigenvalue spectrum of the fluid-solid Jacobian matrix. The latter was obtained by analytically deriving the linearized equations and space-discretizing the Navier-Stokes model with finite elements.

Using this approach, one can capture, with one uniform flow model, various types of fluid-solid instabilities. First, a classical coupled-mode flutter occurred at highenough reduced velocity due to the convergence of the heaving and pitching frequencies. For even higher velocity, a static divergence instability was found. For low reduced velocities, vortex induced vibrations instabilities occur, as the hydrodynamic wake mode interacts with the natural heaving or pitching mode. By varying the angle of attack of the steady solution, we showed how the flutter instability progressively transitions from a coupledmode flutter involving both heaving and pitching motions to a single-mode flutter involving only the pitching mode. This transition is concomitant with the appearance of a 

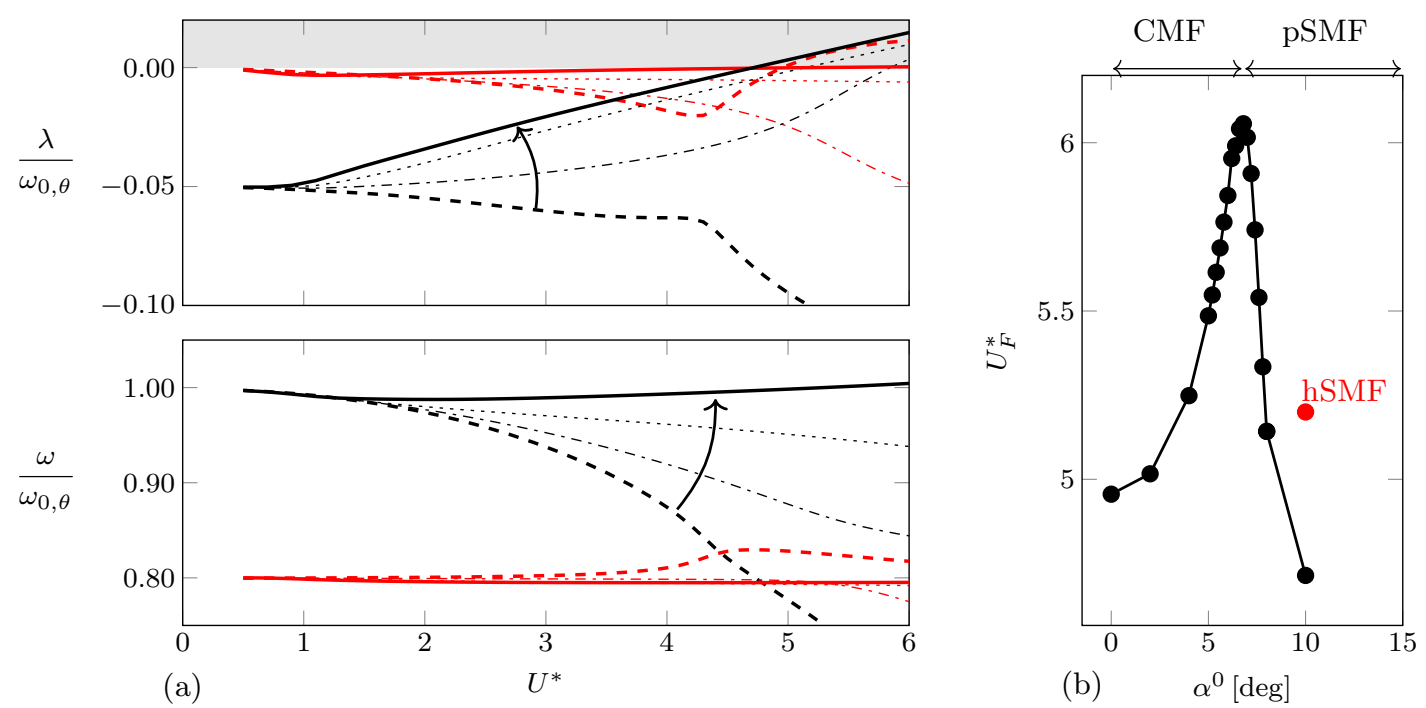

FIG. 12. Transition from Coupled-Mode Flutter (CMF) to pitching Single-Mode Flutter (pSMF) as the steady angle of attack increases $\left(\mathcal{R} e=500, m=10^{3}\right)$. (a) Evolution of the growth rate (top) and frequency (bottom) as a function of the reduced velocity for the pitching (black) and heaving (red) modes and different steady angles of attack : $\alpha^{0}=0^{\circ}$ (dashed), $6^{\circ}$ (dashdotted), $8^{\circ}$ (dotted) and $10^{\circ}$ (solid). (b) Evolution of the critical reduced velocity with the steady angle of attack. When the steady solution is stalled $\left(\alpha^{0}>9^{\circ}\right)$, a heaving Single-Mode Flutter (hSMF) also gets unstable.
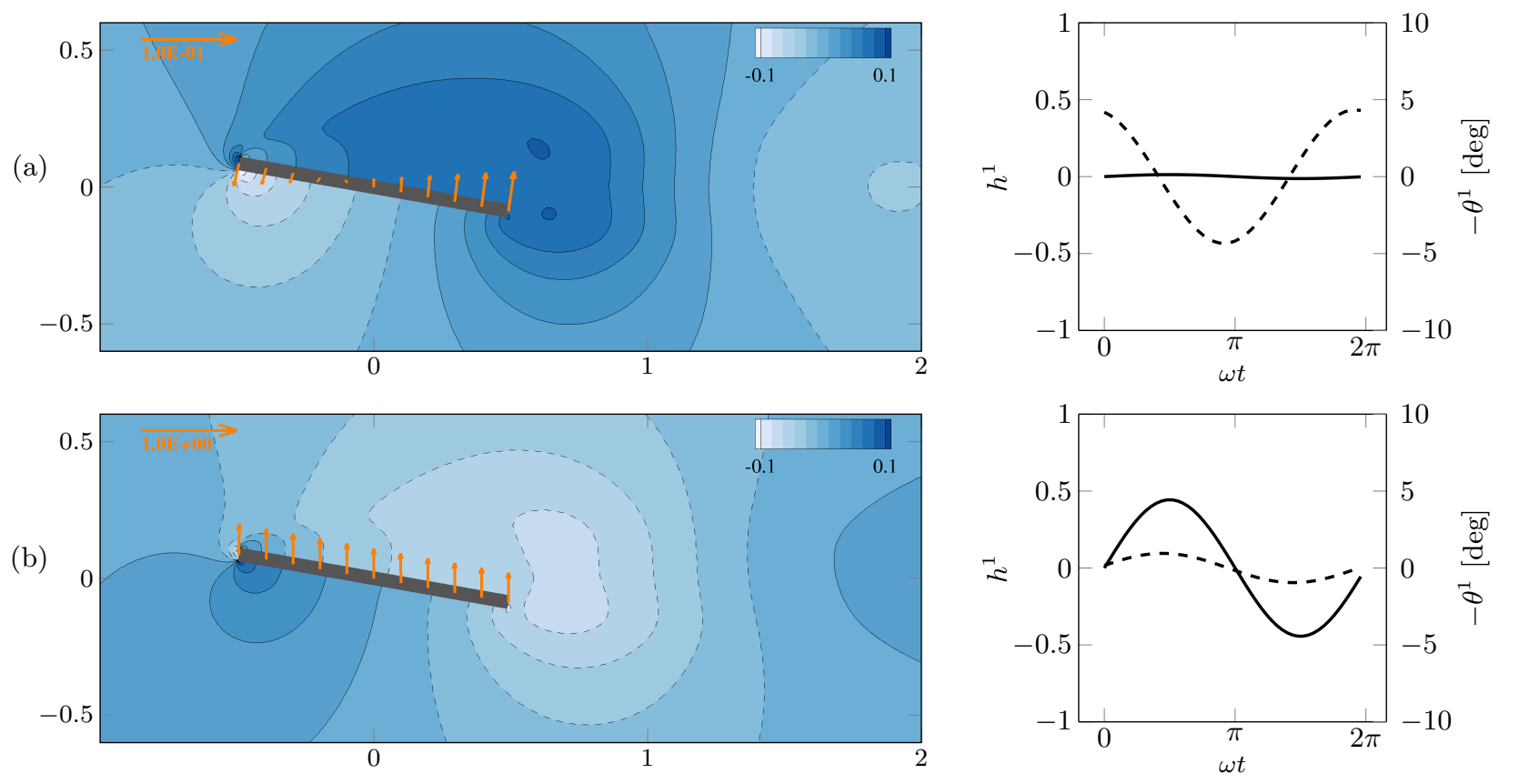

FIG. 13. Critical eigenmodes for (a) $\operatorname{pSMF}\left(U_{F}^{*}=4.8\right)$ and (b) $\operatorname{hSMF}\left(U_{F}^{*}=5.2\right)$ at $\left(\mathcal{R} e=500, m=10^{3}, \alpha^{0}=10^{\circ}\right)$. Color codes and legends are identical to fig. 4.

large recirculation region on the plate suction side.

Having identified these different instabilities, neutral curves were presented in the $\left(m, U^{*}\right)$ plane for the case of zero steady angle of attack. For all mass ratios, vortexinduced vibrations occurred on a range of reduced velocities centered around two well-defined values that correspond to the coincidence of the pitching (resp. heaving) frequencies with the hydrodynamic wake instability frequency. Decreasing the mass ratio tends to widen the range of reduced velocity where vortex-induced vibrations occur. At a particular, low, mass ratio $m^{I I}$ (about $m^{I I} \sim 10$ at $\mathcal{R} e=2900$ ) a codimension-two point is found where both flutter and divergence occur at the same critical reduced velocity. For mass ratios higher 
than $m^{I I}$, flutter occurs prior to divergence whereas divergence precedes flutter for mass ratios below $m^{I I}$. In addition, it was shown that for low mass ratios, slightly below $m^{I I}$, the flutter neutral curve features a turning point. The latter implies the existence of a high- $U^{*}$ flutter boundary above which the flutter mode restabilizes. A study of the effect of the Reynolds number showed that this high- $U^{*}$ restabilization of the flutter mode is delayed by increasing $\mathcal{R} e$, thus leading us to suggest this unexpected behavior is due to viscosity effects. The exact mechanism at play however remains to be precised. The flutter neutral curves obtained from a calibrated version of the Theodorsen model, using viscous steady lift and moment slopes, were compared to the ones obtained with our fully viscous analysis. In the limit of large mass ratios, where the critical flutter frequency is typically low, the calibrated Theodorsen model well predicts the low- $U^{*}$ flutter threshold. However, as the mass ratio decreases, such a model increasingly overestimates the threshold, typically of about $35 \%$ at $m=10$ and $\mathcal{R} e=2900$. In addition, the Theodorsen model is not able to predict the turning of the flutter neutral curve and the consecutive high- $U^{*}$ restabilization observed with global fluid-solid stability. This result further seems to indicate that the high- $U^{*}$ restabilization is linked to unsteady viscous effects, not taken into by a statically calibrated Theodorsen model.

\section{ACKNOWLEDGMENTS}

This project has received funding from the European Research Council (ERC) under the European Union Horizon2020 research and innovation program (grant agreement 638307).

\section{DATA AVAILABILITY}

The data corresponding to the figures and tables of this manuscript are available on request from the author. In addition, the FreeFEM codes used to compute steady solutions, eq. (7) and solve the fluid-solid stability problem, eq. (8) are available at https://bitbucket.org/ jmoulin/pof 2021 .

\section{Appendix A: Definition of the fluid and solid operators in the compact formulations}

In this appendix, we provide the detailed expressions of the fluid and solid operators used in the compact formulation of the evolution equation (6), the steady state equation (7) and the eigenproblem (8), for the solid variable $\mathbf{q}_{\mathrm{s}}=(h, \theta, \dot{h}, \dot{\theta})^{\mathrm{T}}$ and the fluid variable $\mathbf{q}_{\mathrm{f}}=(\mathbf{U}, P)^{\mathrm{T}}$.
The mass matrices are defined as

$$
\mathscr{M}_{\mathrm{S}}=\left(\begin{array}{llll}
1 & 0 & 0 & 0 \\
0 & 1 & 0 & 0 \\
0 & 0 & 1 & 0 \\
0 & 0 & 0 & 1
\end{array}\right) \quad \text { and } \quad \mathscr{M}_{\mathrm{f}}=\left(\begin{array}{ll}
1 & 0 \\
0 & 0
\end{array}\right) .
$$

The solid $\mathscr{R}_{\mathrm{s}}$ and fluid $\mathscr{R}_{\mathrm{f}}$ residual vectors in the nonlinear equations (6) and (7) read:

$$
\begin{aligned}
& \mathscr{R}_{\mathrm{s}}\left(\mathbf{q}_{\mathrm{s}}, \mathbf{q}_{\mathrm{f}}\right)= \\
& \left(\begin{array}{c}
-\dot{h} \\
-\dot{\theta} \\
2 \zeta_{h} \frac{\Omega}{U^{*}} \dot{h}+\frac{\Omega^{2}}{U^{* 2}} h-m^{-1}\left(\mathcal{C}_{L}(\mathbf{U}, P, \theta)+\mathcal{C}_{F_{\mathrm{ext}}}\right) \\
2 \zeta_{\theta} \frac{1}{U^{*}} \dot{\theta}+\frac{1}{U^{* 2}} \theta-m^{-1} r_{\theta}{ }^{-2}\left(\mathcal{C}_{M}(\mathbf{U}, P)+\mathcal{C}_{M_{\mathrm{ext}}}\right)
\end{array}\right), \\
& \mathscr{R}_{\mathrm{f}}\left(\mathbf{q}_{\mathrm{s}}, \mathbf{q}_{\mathrm{f}}\right)= \\
& \left(\begin{array}{c}
\dot{\theta} \mathbf{e}_{\mathbf{z}} \wedge \mathbf{U}+\nabla \mathbf{U}(\mathbf{U}-\mathbf{W}(\theta, \dot{h}, \dot{\theta}))-\nabla \cdot \boldsymbol{\sigma}(\mathbf{U}, P) \\
\nabla \cdot \mathbf{U}
\end{array}\right) .
\end{aligned}
$$

where $\boldsymbol{\sigma}$ is the classical Cauchy stress tensor. For convenience, we recall here the definitions of the lift coefficient $\mathcal{C}_{L}$, the moment coefficient $\mathcal{C}_{M}$ computed at the center of mass $G$ located at $\mathbf{X}=(0,0)$ and of the grid velocity W:

$$
\begin{aligned}
& \mathcal{C}_{L}(\mathbf{U}, P, \theta)= 2\left\{\mathbf{R}(\theta) \int_{\Gamma_{\mathrm{w}}} \boldsymbol{\sigma}(\mathbf{U}, P) \mathbf{N} d \mathbf{X}_{\mathrm{w}}\right\} \cdot \mathbf{e}_{\mathbf{y}} \\
& \mathcal{C}_{M}(\mathbf{U}, P)=2 \int_{\Gamma_{\mathrm{w}}}\left\{X_{\mathrm{w}}(\boldsymbol{\sigma}(\mathbf{U}, P) \mathbf{N})_{Y}\right. \\
&\left.\quad-Y_{\mathrm{w}}(\boldsymbol{\sigma}(\mathbf{U}, P) \mathbf{N})_{X}\right\} d \mathbf{X}_{\mathrm{w}} \\
& \mathbf{W}(\theta, \dot{h}, \dot{\theta} ; \mathbf{X})=\mathbf{R}(\theta)^{\mathrm{T}}(-1, \dot{h})^{\mathrm{T}}+\dot{\theta}\left(\mathbf{e}_{\mathbf{z}} \wedge \mathbf{X}\right)
\end{aligned}
$$

The submatrices in the eigenproblem (8) are obtained by linearization of the solid and fluid residual vectors around the solid $\mathbf{q}_{\mathrm{s}}^{0}$ and fluid $\mathbf{q}_{\mathrm{f}}^{0}$ steady states. For the solid equations, they read:

$$
\begin{aligned}
\mathscr{J}_{\mathrm{ss}}\left(\mathbf{q}_{\mathrm{s}}^{0}, \mathbf{q}_{\mathrm{f}}^{0}\right) & =\left(\begin{array}{cccc}
0 & 0 & -1 & 0 \\
0 & 0 & 0 & -1 \\
\Omega^{2} U^{*-2} & -\mathscr{L}_{\theta} & 2 \zeta_{h} \Omega U^{*-1} & 0 \\
0 & U^{*-2} & 0 & 2 \zeta_{\theta} U^{*-1}
\end{array}\right) \\
\mathscr{J}_{\mathrm{sf}}\left(\mathbf{q}_{\mathrm{s}}^{0}, \mathbf{q}_{\mathrm{f}}^{0}\right) & =\left(\begin{array}{cc}
0 & 0 \\
0 & 0 \\
-\mathscr{L}_{\mathbf{U}} & -\mathscr{L}_{P} \\
-\mathscr{M}_{\mathbf{U}} & -\mathscr{M}_{P}
\end{array}\right)
\end{aligned}
$$

where the terms $\mathscr{L}_{\theta}, \mathscr{L}_{\mathrm{U}}$ and $\mathscr{L}_{P}$ are the derivatives of the lift coefficient $\mathcal{C}_{L}$ defined as:

$$
\mathscr{L}_{\theta}=m^{-1} \frac{\partial \mathcal{C}_{L}}{\partial \theta}, \quad \mathscr{L}_{\mathbf{U}}=m^{-1} \frac{\partial \mathcal{C}_{L}}{\partial \mathbf{U}}, \quad \mathscr{L}_{P}=m^{-1} \frac{\partial \mathcal{C}_{L}}{\partial P}
$$

while $\mathscr{M}_{\mathrm{U}}$ and $\mathscr{M}_{P}$ are the derivatives of the moment coefficient $\mathcal{C}_{M}$ defined as:

$$
\mathscr{M}_{\mathbf{U}}=m^{-1} r_{\theta}{ }^{-2} \frac{\partial \mathcal{C}_{M}}{\partial \mathbf{U}}, \quad \mathscr{M}_{P}=m^{-1} r_{\theta}{ }^{-2} \frac{\partial \mathcal{C}_{M}}{\partial P}
$$


The submatrices for the fluid equations are defined as:

$$
\begin{aligned}
& \mathscr{J}_{\mathrm{fs}}\left(\mathbf{q}_{\mathrm{s}}^{0}, \mathbf{q}_{\mathrm{f}}^{0}\right)=\left(\begin{array}{cccc}
0 & \mathscr{D}_{\theta} & \mathscr{D}_{\dot{h}} & \mathscr{D}_{\dot{\theta}} \\
0 & 0 & 0 & 0
\end{array}\right) \\
& \mathscr{J}_{\mathrm{ff}}\left(\mathbf{q}_{\mathrm{s}}^{0}, \mathbf{q}_{\mathrm{f}}^{0}\right)=\left(\begin{array}{cc}
\mathscr{A} & \nabla \bullet \\
\nabla \cdot \bullet & 0
\end{array}\right) .
\end{aligned}
$$

where $\mathscr{A}$ is a short notation for the derivative of the Navier-Stokes momentum equation with respect to the fluid velocity $\mathbf{U}$ :

$$
\begin{array}{r}
\mathscr{A}=\nabla(\bullet)\left(\mathbf{U}^{0}-\mathbf{W}\left(0, \theta^{0}, 0\right)\right)+\nabla \mathbf{U}^{0} \bullet \\
-\mathcal{R} e^{-1} \nabla \cdot\left(\nabla \bullet+\nabla \bullet^{\mathrm{T}}\right)
\end{array}
$$

The terms $\mathscr{D}_{\theta}, \mathscr{D}_{\dot{h}}$ and $\mathscr{D}_{\dot{\theta}}$ are the so-called shape derivatives, i.e. the derivatives of the Navier-Stokes equations with respect to the solid motion:

$$
\begin{aligned}
& \mathscr{D}_{\theta}=-\nabla \mathbf{U}^{0} \frac{\partial \mathbf{W}}{\partial \theta}, \quad \mathscr{D}_{\dot{h}}=-\nabla \mathbf{U}^{0} \frac{\partial \mathbf{W}}{\partial \dot{h}} \\
& \mathscr{D}_{\dot{\theta}}=-\nabla \mathbf{U}^{0} \frac{\partial \mathbf{W}}{\partial \dot{\theta}}+\mathbf{e}_{\mathbf{z}} \wedge \mathbf{U}^{0}
\end{aligned}
$$

where the derivatives of the grid velocity $\mathbf{W}$ to the solid variables are straightforward to obtain.

\section{Appendix B: Validation: spring-mounted cylinder case}

For validation purposes, our linear stability solver was tested on the configuration consisting in the flow around a circular cylinder mounted on a heaving spring, which has been largely documented in the literature ${ }^{38,40,41}$. To this end, we adapt our set of non-dimensional numbers to the conventions used by Navrose and Mittal ${ }^{40}$, Sabino et al. ${ }^{41}$, yielding:

$$
U^{*}=\frac{2 \pi \tilde{U}_{\infty}}{\tilde{D} \sqrt{\tilde{K}_{h} / \tilde{m}}}, \quad m=\frac{4 \tilde{m}}{\pi \tilde{\rho} \tilde{D}^{2} \tilde{s}}, \quad \mathcal{C}_{L}=\frac{\tilde{L}}{0.5 \tilde{\rho} \tilde{U}_{\infty}^{2} \tilde{D} \tilde{s}}
$$

which gives the following variant of the heaving equation:

$$
\frac{\mathrm{d}^{2} h}{\mathrm{~d} t^{2}}+\left(\frac{2 \pi}{U^{*}}\right)^{2} h=\frac{2}{\pi m} \mathcal{C}_{L}
$$

The pitching spring, for its part, is kept infinitely rigid. For a particular Reynolds number $\mathcal{R} e=60$ and mass ratio $m=20$, we show in fig. 14 the variations of the so-called $^{40}$ fluid (blue) and solid (red) modes as a function of $U^{*}$. Our results, marked by open circles, perfectly match the previous work by Sabino et al. ${ }^{41}$ (dashed lines). In addition, we verify that in the limit of an infinitely rigid heaving spring $\left(U^{*} \rightarrow 0\right)$, the fluid mode (blue mode) tends towards the vortex shedding mode classically obtained ${ }^{66,76-78}$ for a fixed cylinder (horizontal blue lines). These results validate our computational approach by showing it accurately reproduces the classical results for a heaving spring-mounted cylinder undergoing vortex-induced vibrations.

\section{Appendix C: Static calibration of the Theodorsen model}

In the original work by Theodorsen ${ }^{27}$, analytical expressions where derived for the fluid lift and moment applied by a potential flow on an infinitely thin flat plate forced to oscillate in pitch and plunge. Using the particular sign and non-dimensionalization conventions adopted in the present work, these read:

$$
\begin{aligned}
\mathcal{C}_{L}=-\frac{\pi}{2}[\dot{\theta} & +\ddot{h}-a \ddot{\theta}] \\
& +\left.C(k) \frac{\mathrm{d} \mathcal{C}_{L}^{\text {stat }}}{\mathrm{d} \theta}\right|_{\theta_{0}}\left[\theta+\dot{h}+\left(\frac{1}{4}-a\right) \dot{\theta}\right] \\
\mathcal{C}_{M}=\frac{\pi}{4}[a \ddot{h} & \left.-\left(\frac{1}{16}+2 a^{2}\right) \ddot{\theta}\right]+\frac{\pi}{4}\left(2 a-\frac{1}{2}\right) \dot{\theta} \\
& +\left.C(k) \frac{\mathrm{d} \mathcal{C}_{M}^{\text {stat }}}{\mathrm{d} \theta}\right|_{\theta_{0}}\left[\theta+\dot{h}+\left(\frac{1}{4}-a\right) \dot{\theta}\right]
\end{aligned}
$$

where $a$ is the nondimensional distance between the elastic axis and the mid-chord (counted positively when the elastic axis is downstream of the mid-chord) - here $a=0$ - , and where $C(k=\omega / 2)$ is the Theodorsen function, which can be analytically expressed using Hankel's functions. In particular, the rightmost terms in eq. (C1) involve the steady lift and moment slopes at a steady pitching angle $\theta^{0}, \mathrm{~d} \mathcal{C}_{L}^{\text {stat }} /\left.\mathrm{d} \theta\right|_{\theta_{0}}$ and $\mathrm{d} \mathcal{C}_{M}^{\text {stat }} /\left.\mathrm{d} \theta\right|_{\theta_{0}}$. In the original theory, the thin airfoil theory values are naturally substituted for the lift and moment slopes:

$$
\left.\frac{\mathrm{d} \mathcal{C}_{L}^{\text {stat }}}{\mathrm{d} \theta}\right|_{\theta_{0}}=-\left.2 \pi \quad \frac{\mathrm{d} \mathcal{C}_{M}^{\text {stat }}}{\mathrm{d} \theta}\right|_{\theta_{0}}=\frac{\pi}{2}(1+4 a)
$$

In the present work, we use instead a simple calibration strategy which consists in replacing the above by their viscous counterparts that can be either obtained experimentally ${ }^{14}$ or, in our case, computed numerically. Indeed, by differentiating the lift and moment coefficients, eq. (5), with respect to $\theta$, we obtain:

$$
\begin{array}{lll}
\left.\frac{\mathrm{d} \mathcal{C}_{L}^{\text {stat }}}{\mathrm{d} \theta}\right|_{\theta^{0}}=\left.\frac{\partial \mathcal{C}_{L}}{\partial \theta}\right|_{0} & +\left.\frac{\partial \mathcal{C}_{L}}{\partial \mathbf{U}}\right|_{0} \frac{\partial \mathbf{U}^{0}}{\partial \theta} & +\left.\frac{\partial \mathcal{C}_{L}}{\partial P}\right|_{0} \frac{\partial P^{0}}{\partial \theta} \\
\left.\frac{\mathrm{d} \mathcal{C}_{M}^{\text {stat }}}{\mathrm{d} \theta}\right|_{\theta^{0}}=0 & +\left.\frac{\partial \mathcal{C}_{M}}{\partial \mathbf{U}}\right|_{0} \frac{\partial \mathbf{U}^{0}}{\partial \theta} & +\left.\frac{\partial \mathcal{C}_{M}}{\partial P}\right|_{0} \frac{\partial P^{0}}{\partial \theta}
\end{array}
$$

The only unknown quantities in (C2) are the steady fluid velocity and pressure increments, $\partial \mathbf{U}^{0} / \partial \theta$ and $\partial P^{0} / \partial \theta$, due to an unit variation in $\theta$. A linear problem governing these quantities can be derived by differentiating the steady-state Navier-Stokes equations with respect to $\theta$, which yields:

$$
\left(\begin{array}{cc}
\mathscr{A} & \nabla \bullet \\
\nabla \cdot \bullet & 0
\end{array}\right)\left(\begin{array}{c}
\partial \mathbf{U}^{0} / \partial \theta \\
\partial P^{0} / \partial \theta
\end{array}\right)=\left(\begin{array}{c}
-\mathscr{D}_{\theta} \\
0
\end{array}\right)
$$

where the operators $\mathscr{A}$ and $\mathscr{D}_{\theta}$ have been previously defined in appendix A. As a consequence, the steady slopes of the lift and moment coefficients at $\theta^{0}$ can be obtained 


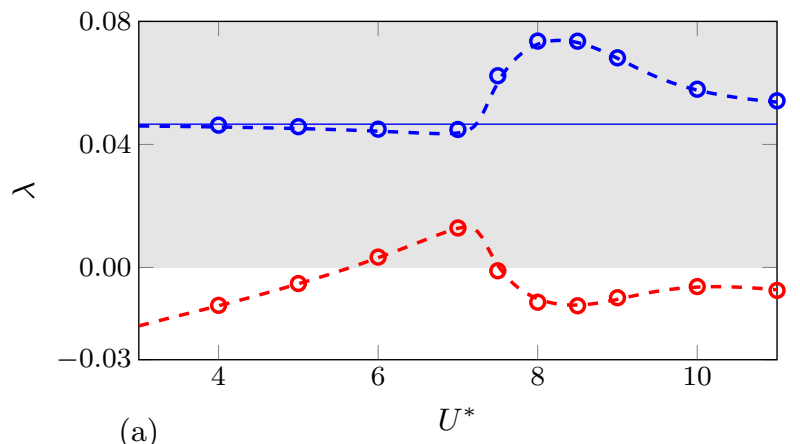

(a)

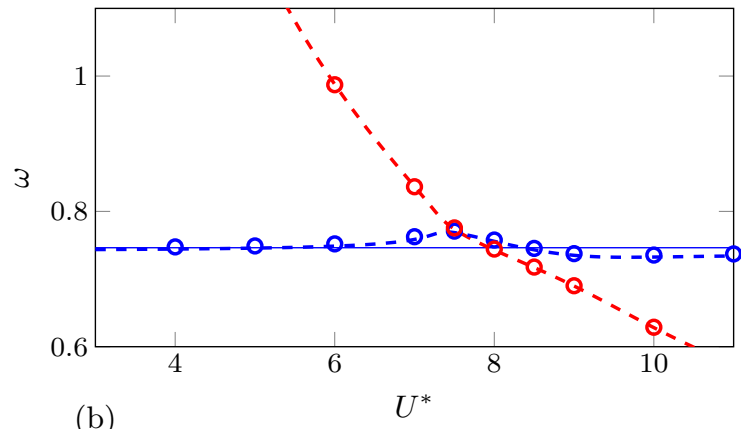

(b)

FIG. 14. Validation of the linear stability analysis solver on the spring-mounted cylinder flow $(\mathcal{R} e=60, m=20)$. The variation with $U^{*}$ of the growth rate (a) and frequency (b) of the so-called ${ }^{40}$ fluid (blue circles) and solid (red circles) modes are compared with the results of Sabino et al. ${ }^{41}$ (dashed). The thin horizontal blue lines materialize the vortex shedding mode of the fixed cylinder case.

from the steady-state solution at $\theta^{0}$ at the (only) additional cost of solving the above linear problem.

For a range of Reynolds numbers between 10 and $10^{4}$ we show in fig. 15 the values of the lift (a) an moment (b) slopes obtained through eq. (C2), showing significant variations away from the thin airfoil theory values materialized by the thin horizontal lines. Two additional remarks must be made. First, it is remarkable that here the thin airfoil theory turns out to fairly predict the flow at a Reynolds close to the critical Reynolds for the wake instability on the fixed plate, $\mathcal{R} e_{c \text {,wake }}$ (vertical dashed line). It would be interesting to investigate whether this is pure coincidence, or a more broadly observed feature. Second, one must take the large slopes obtained for

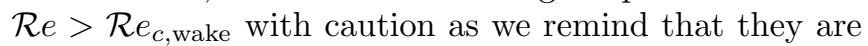
computed from steady Navier-Stokes solutions which are unstable at those high Reynolds numbers.

In fig. 15(c) we compare the critical flutter velocity predicted by the global stability approach (solid line) and the calibrated Theodorsen model (dashed line with square symbols) for $10 \leq \mathcal{R} e \leq 10^{4}$ and a mass ratio $m=10^{4}$. For such a large mass ratio, the calibration yields a good agreement between both the Theodorsen model and the global stability results. In contrast, the non-calibrated Theodorsen theory (thin horizontal line) is only valid for the particular Reynolds number, that happens to present steady slopes close to the thin airfoil theory predictions. As a consequence, for a large mass ratio such as $m=10^{4}$, calibrating the Theodorsen model with steady viscous slopes is well-adapted to capture the variation of the critical velocity due to varying the Reynolds number. This not true anymore for lower mass ratios, as seen in fig. 10 .

\footnotetext{
${ }^{1}$ R. L. Bisplinghoff, H. Ashley, and R. L. Halfman, Aeroelasticity (1955).

${ }^{2}$ E. H. Dowell, H. C. Curtiss, R. H. Scanlan, and F. Sisto, A modern Course in Aeroelasticity, springer ed. (1989).

${ }^{3}$ F. Richez, M. Leguille, and O. Marquet, "Selective frequency damping method for steady RANS solutions of turbulent separated flows around an airfoil at stall," Computers and Fluids 132, 51-61 (2016).

${ }^{4}$ D. Busquet, O. Marquet, F. Richez, M. Juniper, and D. Sipp,
}

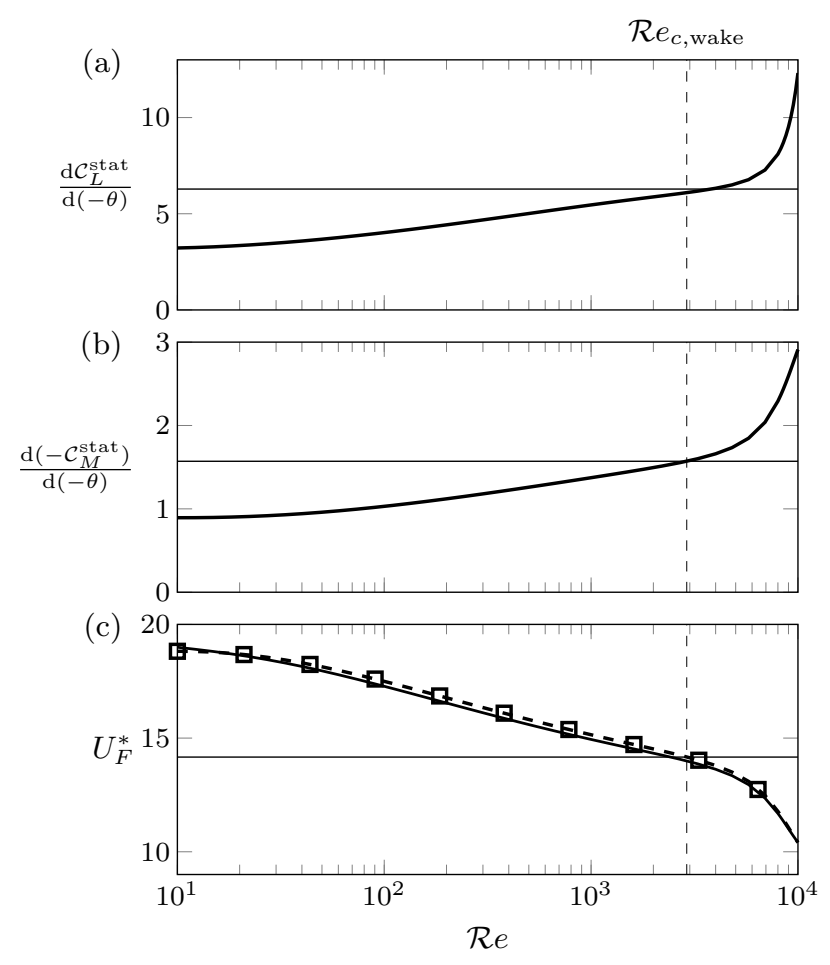

FIG. 15. Calibration of the Theodorsen model with the slopes of the steady $\mathcal{C}_{L}^{\text {stat }}(\theta)$ and $\mathcal{C}_{M}^{\text {stat }}(\theta)$ curves, evaluated at $\theta_{0}=$ 0 . The slopes of the lift and moment coefficients are shown as a function of $\mathcal{R} e$ in (a) and (b) respectively. In (c), we compare the critical flutter velocity at $m=10^{4}$ obtained with full Navier-Stokes (solid line) and the statically calibrated Theodorsen model (dashed line with square symbols). The thin horizontal lines shows the potential flow $(\mathrm{a}, \mathrm{b})$ or noncalibrated Theodorsen theory (c) values. The vertical dashed line materializes the critical Reynolds for the wake instability.

"Global stability analysis of turbulent flows around an airfoil near stall," in Eurogen 2017, September (Madrid, 2017).

${ }^{5}$ R. L. Halfman, H. C. Johnson, and S. M. Haley, "Evaluation of high-angle-of-attack aerodynamic-derivative data and stallflutter prediction techniques," Tech. Rep. (NATIONAL AERO- 
NAUTICS AND SPACE ADMINISTRATION WASHINGTON DC, 1951).

${ }^{6}$ N. A. Razak, T. Andrianne, and G. Dimitriadis, "Flutter and Stall Flutter of a Rectangular Wing in a Wind Tunnel," AIAA Journal 49, 2258-2271 (2011).

${ }^{7}$ S. S. Bhat and R. N. Govardhan, "Stall flutter of NACA 0012 airfoil at low Reynolds numbers," Journal of Fluids and Structures 41, 166-174 (2013).

${ }^{8}$ C. H. Williamson and R. Govardhan, "Vortex-induced vibrations," Annual Review of Fluid Mechanics 36, 413-455 (2004).

${ }^{9}$ A. B. Rostami and M. Armandei, "Renewable energy harvesting by vortex-induced motions: Review and benchmarking of technologies," Renewable and Sustainable Energy Reviews 70, 193-214 (2017).

${ }^{10} \mathrm{~A}$. Rips and R. Mittal, "Enhanced mixing at inertial microscales using flow-induced flutter," Phys. Rev. Fluids 4, 54501 (2019).

${ }^{11}$ A. Rips and R. Mittal, "Flutter-enhanced mixing in small-scale mixers," Physics of Fluids 31 (2019), 10.1063/1.5115351.

${ }^{12}$ K. Shoele and R. Mittal, "Computational study of flow-induced vibration of a reed in a channel and effect on convective heat transfer," 127103, 1-25 (2014).

${ }^{13}$ A. Rips, K. Shoele, A. Glezer, and R. Mittal, "Efficient Electronic Cooling via Flow-Induced Vibrations," in 33rd Thermal Measurement, Modelling \& Management Symposium (SEMITHERM) (2017).

${ }^{14}$ X. Amandolese, S. Michelin, and M. Choquel, "Low speed flutter and limit cycle oscillations of a two-degree-of-freedom flat plate in a wind tunnel," Journal of Fluids and Structures 43, 244-255 (2013).

${ }^{15}$ L. Pigolotti, C. Mannini, G. Bartoli, and K. Thiele, "Wind tunnel tests on elongated rectangular plates under flutter motion: limit-cycle oscillations and preliminary energy harvesting considerations," in 14th International Conference on Wind Engineering (Porto Alegre, Brazil, 2015).

${ }^{16}$ K. Onoue, A. Song, B. Strom, and K. S. Breuer, "Large amplitude flow-induced oscillations and energy harvesting using a cyber-physical pitching plate," Journal of Fluids and Structures 55, 262-275 (2015).

${ }^{17} \mathrm{~K}$. Onoue and K. S. Breuer, "Vortex formation and shedding from a cyber-physical pitching plate," Journal of Fluid Mechanics 793, 229-247 (2016).

${ }^{18}$ S. Olivieri, G. Boccalero, A. Mazzino, and C. Boragno, "Fluttering conditions of an energy harvester for autonomous powering," Renewable Energy 105, 530-538 (2017).

${ }^{19}$ A. Malher, O. Doaré, and C. Touzé, "Influence of a hysteretic damper on the flutter instability," Journal of Fluids and Structures 68, 356-369 (2017).

${ }^{20}$ M. Boudreau, G. Dumas, M. Rahimpour, and P. Oshkai, "Experimental investigation of the energy extraction by a fully-passive flapping-foil hydrokinetic turbine prototype," Journal of Fluids and Structures 82, 446-472 (2018).

${ }^{21}$ Z. Peng and Q. Zhu, "Energy harvesting through flow-induced oscillations of a foil," Physics of Fluids 21, 1-9 (2009).

${ }^{22}$ A. Orchini, A. Mazzino, J. Guerrero, R. Festa, and C. Boragno, "Flapping states of an elastically anchored plate in a uniform flow with applications to energy harvesting by fluid-structure interaction," Physics of Fluids 25 (2013), 10.1063/1.4821808.

${ }^{23}$ Z. Wang, L. Du, J. Zhao, and X. Sun, "Structural response and energy extraction of a fully passive flapping foil," Journal of Fluids and Structures 72, 96-113 (2017).

${ }^{24} \mathrm{~K}$. Menon and R. Mittal, "Flow physics and dynamics of flowinduced pitch oscillations of an airfoil," Journal of Fluid Mechanics 877, 582-613 (2019).

${ }^{25}$ Z. Wang, L. Du, J. Zhao, M. C. Thompson, and X. Sun, "Flowinduced vibrations of a pitching and plunging airfoil," Journal of Fluid Mechanics 885 (2020), 10.1017/jfm.2019.996.

${ }^{26} \mathrm{~K}$. Menon and R. Mittal, "Dynamic mode decomposition based analysis of flow over a sinusoidally pitching airfoil," Journal of Fluids and Structures 94, 102886 (2020), arXiv:1911.05768.

${ }^{27} \mathrm{~T}$. Theodorsen, "General Theory of Aerodynamic Instability and the Mechanism of Flutter," Tech. Rep. (NACA Report 496, Langley, 1935).

${ }^{28}$ E. Garrigues, "A Review of Industrial Aeroelasticity Practices at Dassault Aviation for Military Aircraft and Business Jets," AerospaceLab Journal , 1-34 (2018).

${ }^{29}$ L. Bruno and D. Fransos, "Evaluation of Reynolds number effects on flutter derivatives of a flat plate by means of a computational approach," Journal of Fluids and Structures 24, 1058-1076 (2008).

${ }^{30}$ S. L. Brunton and C. W. Rowley, "Empirical state-space representations for Theodorsen's lift model," Journal of Fluids and Structures 38, 174-186 (2013).

${ }^{31}$ W. Shyy, H. Aono, S. K. Chimakurthi, P. Trizila, C. K. Kang, C. E. Cesnik, and H. Liu, "Recent progress in flapping wing aerodynamics and aeroelasticity," Progress in Aerospace Sciences 46, 284-327 (2010).

${ }^{32}$ M. P. Païdoussis, S. J. Price, and E. De Langre, Fluid Structure Interactions : Cross-Flow-Induced Instabilities, cambridge ed. (New York, 2011).

${ }^{33}$ M. L. Facchinetti, E. de Langre, and F. Biolley, "Coupling of structure and wake oscillators in vortex-induced vibrations," Journal of Fluids and Structures 19, 123-140 (2004).

${ }^{34} \mathrm{E}$. de Langre, "Frequency lock-in is caused by coupled-mode flutter," Journal of Fluids and Structures 22, 783-791 (2006).

${ }^{35}$ S. P. Singh and S. Mittal, "Vortex-induced oscillations at low reynolds numbers: Hysteresis and vortex-shedding modes," Journal of Fluids and Structures 20, 1085-1104 (2005).

${ }^{36}$ D. Sipp, O. Marquet, P. Meliga, and A. Barbagallo, "Dynamics and Control of Global Instabilities in Open-Flows: A Linearized Approach," Applied Mechanics Reviews 63 (2010), $10.1115 / 1.4001478$.

${ }^{37}$ V. Theofilis, "Global Linear Instability," Annual Review of Fluid Mechanics 43, 319-352 (2011).

${ }^{38} \mathrm{C}$. Cossu and L. Morino, "On the Instability of a Spring-Mounted Circular Cylinder in a Viscous Flow at Low Reynolds Numbers," Journal of Fluids and Structures 14, 183-196 (2000).

${ }^{39}$ S. Mittal and S. Singh, "Vortex-induced vibrations at subcritical Re," Journal of Fluid Mechanics 534, 185-194 (2005).

${ }^{40}$ Navrose and S. Mittal, "Lock-in in vortex-induced vibration," J. Fluid Mech 794, 565-594 (2016).

${ }^{41}$ D. Sabino, D. Fabre, J. S. Leontini, and D. Lo Jacono, "Vortexinduced vibration prediction via an impedance criterion," Journal of Fluid Mechanics 890 (2020), 10.1017/jfm.2020.104.

${ }^{42}$ C. Gao, W. Zhang, X. Li, Y. Liu, J. Quan, Z. Ye, and Y. Jiang, "Mechanism of frequency lock-in in transonic buffeting flow," Journal of Fluid Mechanics 818, 528-561 (2017).

${ }^{43}$ C. Gao and W. Zhang, "Transonic aeroelasticity: A new perspective from the fluid mode," Progress in Aerospace Sciences , 100596 (2020).

${ }^{44}$ P. Assemat, D. Fabre, and J. Magnaudet, "The onset of unsteadiness of two-dimensional bodies falling or rising freely in a viscous fluid: A linear study," Journal of Fluid Mechanics 690, 173-202 (2012).

${ }^{45}$ J. Tchoufag, D. Fabre, and J. Magnaudet, "Global linear stability analysis of the wake and path of buoyancy-driven disks and thin cylinders," Journal of Fluid Mechanics 740, 278-311 (2014).

${ }^{46} \mathrm{~J}$. Tchoufag, J. Magnaudet, and D. Fabre, "Linear instability of the path of a freely rising spheroidal bubble," Journal of Fluid Mechanics 751, 1-12 (2014).

${ }^{47}$ P. S. Negi, Stability and transition in pitching wings, Ph.D. thesis, Royal Institute of Technology in Stockholm (2019).

${ }^{48}$ A. Goza, T. Colonius, and J. E. Sader, "Global modes and nonlinear analysis of inverted-flag flapping," Journal of Fluid Mechanics 857, 312-344 (2018), arXiv:1709.09745.

${ }^{49}$ J.-L. Pfister, O. Marquet, and M. Carini, "Linear stability analysis of strongly coupled fluid-structure problems with the Arbitrary-Lagrangian-Eulerian method," Computer Methods in Applied Mechanics and Engineering 355, 663-689 (2019).

${ }^{50}$ J. L. Pfister and O. Marquet, "Fluid-structure stability analyses and nonlinear dynamics of flexible splitter plates interacting 
with a circular cylinder flow," Journal of Fluid Mechanics , 1-38 (2020).

${ }^{51}$ J. Houtman and S. Timme, "Towards Global Stability Analysis of Flexible Aircraft in Edge-of-the-Envelope Flow," in AIAA Scitech 2021 Forum (2021).

${ }^{52}$ L. Benetti Ramos, O. Marquet, M. Bergmann, and A. Iollo, "Fluid-solid Floquet stability analysis of self-propelled heaving foils," (2020).

${ }^{53}$ D. Poirel, Y. Harris, and A. Benaissa, "Self-sustained aeroelastic oscillations of a NACA0012 airfoil at low-to-moderate Reynolds numbers," Journal of Fluids and Structures 24, 700-719 (2008).

${ }^{54} \mathrm{D}$. Poirel and F. Mendes, "Experimental Investigation of Small Amplitude Self- Sustained Pitch-Heave Oscillations of a NACA0012 Airfoil at Transitional Reynolds Numbers," in 50th AIAA Aerospace Sciences Meeting including the New Horizons Forum and Aerospace Exposition (Nashville, 2012) pp. 2012-40.

${ }^{55}$ C. J. Barnes and M. R. Visbal, "On the role of flow transition in laminar separation flutter," Journal of Fluids and Structures 77, 213-230 (2018).

${ }^{56}$ D. S. Woolston and G. E. Castile, "Some effects of variations in several parameters including fluid density on the flutter speed of light uniform cantilever wings," Tech. Rep. (NACA Technical Note 2558, 1951).

${ }^{57}$ G. Mougin and J. Magnaudet, "The generalized Kirchhoff equations and their application to the interaction between a rigid body and an arbitrary time-dependent viscous flow," International Journal of Multiphase Flow 28, 1837-1851 (2002).

${ }^{58}$ J. Donea, A. Huerta, J.-P. Ponthot, and A. Rodríguez-Ferran, "Arbitrary Lagrangian-Eulerian Methods," in Encyclopedia of Computational Mechanics (2004) Chap. 14

${ }^{59}$ W. J. Govaerts, Numerical methods for bifurcations of dynamical equilibria (SIAM, 2000).

${ }^{60}$ A. N. Brooks and T. J. R. Hughes, "Streamline upwind/PetrovGalerkin formulations for convection dominated flows with particular emphasis on the incompressible Navier-Stokes equations," Computer Methods in Applied Mechanics and Engineering 32, 199-259 (1982).

${ }^{61}$ F. Hecht, "New development in FreeFem++," J. Numer. Math. 20, 251-265 (2012).

${ }^{62}$ J. Moulin, P. Jolivet, and O. Marquet, "Augmented Lagrangian Preconditioner for Large-Scale Hydrodynamic Stability Analysis," Computer Methods in Applied Mechanics and Engineering (2019).

${ }^{63}$ P. Amestoy, I. Duff, J.-Y. L'Excellent, and J. Koster, "A fully asynchronous multifrontal solver using distributed dynamic scheduling," SIAM Journal on Matrix Analysis and Applications 23, 15-41 (2001).

${ }^{64}$ R. L. Bisplinghoff and H. Ashley, Principles of Aeroelasticity, 2nd ed. (Dover Publications, 1975).

${ }^{65}$ E. De Langre, Fluides et solides (Editions Ecole Polytechnique, 2002).

${ }^{66}$ C. P. Jackson, "A finite-element study of the onset of vortex shedding in flow past variously shaped bodies," Journal of Fluid Mechanics 182, 23-45 (1987).

${ }^{67}$ D. Sipp and A. Lebedev, "Global stability of base and mean flows: A general approach and its applications to cylinder and open cavity flows," Journal of Fluid Mechanics 593, 333-358 (2007).

${ }^{68} \mathrm{O}$. Marquet and M. Larsson, "Global wake instabilities of low aspect-ratio flat-plates," European Journal of Mechanics B/Fluids 49, 400-412 (2015).

${ }^{69}$ C. W. Knisely, "Strouhal numbers of rectangular cylinders at incidence: A review and new data," Journal of Fluids and Structures 4, 371-393 (1990).

${ }^{70}$ S. Ozono, Y. Ohya, Y. Nakamura, and R. Nakayama, "Stepwise increase in the Strouhal number for flows around flat plates," International Journal for Numerical Methods in Fluids 15, 10251036 (1992)

${ }^{71}$ K. Hourigan, M. Thompson, and B. Tan, "Self-sustained oscillations in flows around long blunt plates," Journal of Fluids and
Structures 15, 387-398 (2001)

${ }^{72}$ P. Meliga and J. M. Chomaz, Journal of Fluid Mechanics, Vol. 671 (2011) pp. 137-167.

${ }^{73}$ E. J. Chae, D. T. Akcabay, and Y. L. Young, "Dynamic response and stability of a flapping foil in a dense and viscous fluid," Physics of Fluids 25 (2013), 10.1063/1.4825136.

${ }^{74}$ E. J. Chae, D. T. Akcabay, A. Lelong, J. A. Astolfi, and Y. L. Young, "Numerical and experimental investigation of natural flow-induced vibrations of flexible hydrofoils," Physics of Fluids 28, 075102 (2016).

${ }^{75}$ M. Mahbub Alam, Y. Zhou, H. X. Yang, H. Guo, and J. Mi, "The ultra-low Reynolds number airfoil wake," Experiments in Fluids 48, 81-103 (2009).

${ }^{76}$ F. Gianetti and P. Luchini, "Structural sensitivity of the first instability of the cylinder wake," Journal of Fluid Mechanics 581, 167-197 (2007).

${ }^{77}$ O. Marquet, D. Sipp, and L. Jacquin, "Sensitivity analysis and passive control of cylinder flow," Journal of Fluid Mechanics (2008), 10.1017/S0022112008003662.

${ }^{78}$ D. Fabre, V. Citro, D. Ferreira Sabino, P. Bonnefis, J. Sierra, F. Giannetti, and M. Pigou, "A Practical Review on Linear and Nonlinear Global Approaches to Flow Instabilities," Applied Mechanics Reviews 70, 060802 (2018). 